\title{
article
}

\section{Lamination and folding in electromagnetically driven flows of specified geometries}

\author{
Lionel Rossia* and Sylvain Lardeau ${ }^{\mathrm{a}}$ \\ amperial College London \\ (Received 00 Month 200x; final version received 00 Month 200x)
}

\begin{abstract}
In this paper, a new characterization and a quantification of lamination are presented. The lamination is identified by tracking material lines and by the estimation of the ratio between the length of the material lines within circles and the diameter of the circles. The quantification of the lamination rate relies on the interlaced structure of velocity and Lagrangian acceleration, which allows the determination of the spatial variation of the Lagrangian angular velocity. Those definitions are illustrated using quasi-steady flows driven by electromagnetic forces using mono- and multi-scale configurations with turbulent-like properties. Both experimental and numerical data are used to compute folding rate intensities and lamination for a large range of length scales. Good agreement is found between grid deformation and the prediction of lamination rate, an agreement further confirmed by the measure of lamination. The quantification of lamination is simple and well defined at chosen length-scales. Also, the present form applies to any lines and can be extended to 3D flows. The lamination rate process rely on physical quantities which scale according to the structures' length-scales. It is shown that the lamination rate increases with the reduction of the length-scales and in particular it is found that $\dot{R}_{f o l} \sim \ell^{-3 / 4}$. In turbulent flows, the lamination should then be faster within small coherent structures. This quantification of lamination rate, complemented by a measure of lamination opens new routes for the description and the quantification of mixing in complex and turbulent flows.
\end{abstract}

\section{Introduction}

Osborne Reynolds, in his seminal talk [1], compared mixing in flows with a baker stretching and folding pastry. It is since well known that combinations of stretching and folding can significantly increase flow mixing. This is illustrated on Fig. 1, which shows the evolution of coloured strip (material line) for several stretchinglaminating cycles in a simple flow. During each cycle, the material line $(n=1$, red band, 0$)$ is first stretched by the mean shear $(n=1$, yellow band, 1$)$ and then laminated by the swirling flow $(n=1$, black band, 2$)$. After three cycles, the material black line is striated, i.e. a line crossing this structure will cross alternated layers of black and white. After only five cycles, a dramatic increase in striations and material line length is observed in Fig. 1. Lamination and shear, use independantly, lead to a linear growth of the line length and the striation; when combined, the increase is exponential. This is usually illustrated by the baker process given in Fig. 2 [2]: first, the black strip is stretched to double its size, then it is cut in two equal pieces and both parts are stacked, forming alternated layers of black material strips and the white surrounding. The repetition of this process is the key factor to the exponential growth.

While processes producing fast and local exponential stretching are relatively well-known and characterised (e.g. finite-strain maps, finite-time, local Lyapunov

\footnotetext{
* Corresponding author. Email: l.rossi@imperial.ac.uk or rossi_lionel@yahoo.fr
} 
exponents, hyperbolic stagnation points, [3-7]), the quantification of lamination and folding is still largely unexplored.

Lamination is defined as the ensemble of processes which builds striated structures. Such structures can then be expended before being laminated again, hence generating a dramatic increase of interfaces length. In Fig. 2, this corresponds to the cut-and-stack. Also, folding, which is part of laminating processes, is often related to the need for stretched flow structures to remain in a confined domain [8].

Recently, Bajer et al. [9] showed that, due to differential rotation, the lamination increases the diffusion in the centre of analytical vortices. Accordingly, the analytical whorl illustrated in Fig.3 highlights a higher lamination where the derivative of the angular velocity is the highest. In addition, once laminated structures are exposed to high strain, they are stretched and compressed. In Fig. 4, a striated structure initially represented with four light-grey bands is compressed and stretched within a pure strain region, extending the length of the interface, hence enhancing mixing. During this process, the distance between the striations can be dramatically reduced, leading to an increase in concentration gradients and diffusion between the different layers.

The control of lamination and folding is crucial to improve mixers both at low and high Reynolds number. In complex and turbulent flows, laminated structures can be observed within and around coherent eddies. Once such coherent eddies are merged and/or broken, the laminated domain is stretched. This can then either contribute to a local increase of mixing or be part of a multi-stage baker-like process. More generally, this can occur during the evolution of scalar pattern within flow regions which present topological changes in time (e.g. from elliptic to hyperbolic in Okubo-Weiss partition, [8, 10, 11], see also [12] for three-dimensional flows). The description of these processes is still largely unexplored in turbulent flows. This may be due to the absence of an accepted definition of lamination within flows and to the high computing cost associated with the characterisation of material elements within complex flows. Nevertheless, the measure and the prediction of lamination and folding are important for the fundamental analysis of stirring and mixing.

To date the characterization of lamination and folding often relies on qualitative visualisations [13-16]. In this paper, we introduce a measure of lamination and a quantification of the lamination rate process. This process and measure are then quantified and illustrated using canonical flows driven by mono- and multi-scale electromagnetic forcing [17]. The aim is to provide a basic analytical description of the mixing process of turbulent flows, based on few assumptions. The first one is that any turbulent flow has some persistent geometrical patterns that contribute to mixing. Indeed, the contribution to turbulent mixing of coherent structures and associated saddle points has been highlighted by previous authors, e.g. [18, 19]. The temporal persistence of such geometrical pattern and coherent structures has also been the focus of many studies, e.g. [20-24]. The second assumption is that lamination and folding mechanism occurring in complex flows can be studied using a complementary set of basic flow features. Finally, we assume that canonical patterns persist during their typical turn-over time, which will be considered as a long time-scale reference to characterise lamination.

Two basic flow configurations, complying with the above assumptions, are being investigated in this paper. The first set corresponds to a single scale of forcing. By varying the angle of forcing, different flow geometries can be generated: a pure strain region surrounded by four recirculating domains, a "cat's eyes" flow and an eddy-like recirculation. The second set corresponds to a multi-scale forcing con- 
figurations which reproduces flows with eddies-within-eddies, with turbulent-like properties: the particular arrangement of the magnets leads to a power-law energy spectrum $E(k) \sim k^{-p}$, with $p$ prescribed by the specific arrangement [25, 26], and a Richardson-like diffusion [27], driven by the multi-scale distribution of high stretching regions, with the mean square separation of pairs of fluid element evolving like $\Delta^{2} \sim t^{3}[5]$.

\section{Quantification of lamination and lamination rate}

The terms lamination and lamination rate should be understood in broad sense. The lamination corresponds to the effective striation of material lines. The lamination rate represents the rate at which a material line is bent and/or rolled so as to generate striations. To the author knowledge, it is the first characterisation of lamination rate within flows. This description relies on physical quantities driving the flow dynamics. The measure of lamination, proposed in section 2.2, goes beyond the measure of material line curvatures, e.g. [28], and differs from previous methods, like Horseshoe maps [29] or the geometrical quantification of the interface pattern [30]. The current definition is more straightforward and computationally efficient, and it defines precisely a length-scale of the measure which is independent of the material line.

\subsection{Laminating process}

The Lagrangian acceleration, a, represents the force exerted on a fluid-element (particle fluid) by its surrounding. It governs the alteration in direction and intensity of the Lagrangian velocity, $\mathbf{u}_{\mathbf{L}}$, associated to this fluid-element. The Navier Stokes equations, Eq. (1), describe the spatial and temporal evolution of the Lagrangian acceleration and flow velocity, $\mathbf{u}$, at the spatial position $\mathbf{x}$. The three terms on the r.h.s. of Eq. 1 represent the action of pressure, viscous and body forces, respectively.

$$
\mathbf{a}=\frac{\partial \mathbf{u}_{\mathbf{L}}}{\partial t}=\frac{\partial \mathbf{u}}{\partial t}+(\mathbf{u} \cdot \nabla) \mathbf{u}=-\frac{1}{\rho} \nabla P+\nu \Delta \mathbf{u}+\frac{1}{\rho} \mathbf{f}
$$

At time $t$, the Lagrangian velocity of a fluid-element is equal to the flow velocity at the position of the fluid-element, i.e. $\mathbf{u}_{\mathbf{L}}(t)=\mathbf{u}\left(\mathbf{x}_{\mathbf{E}}, t\right)$, where $\mathbf{x}_{\mathbf{E}}$ is the spatial location of the fluid element. The notation $\mathbf{u}\left(\mathbf{x}_{\mathbf{E}}, t\right)$ will be simplified to $\mathbf{u}$ and the flow is considered as being constituted of an infinite number of fluid elements.

The definition of the lamination rate is based on the ability of the resultant forces to bend and roll a material line at different angular velocities. For this, we consider the alignment between the velocity vector and the Lagrangian acceleration. More precisely, we use the component of the acceleration $\mathbf{a}_{\mathbf{p}}$ perpendicular to the velocity, to determine the local Lagrangian angular velocity, $\dot{\theta}$, i.e. the rate of change of direction of the Lagrangian velocity vector at position $\mathbf{x}$ around the local rotation vector, $\mathbf{e}_{\mathbf{f}}$. These terms are defined as:

$$
\begin{gathered}
\mathbf{a}_{\mathbf{p}}=\mathbf{a}-\mathbf{a}_{\mathbf{t}} ; \mathbf{a}_{\mathbf{t}}=\frac{(\mathbf{u} \cdot \mathbf{a}) \mathbf{u}}{\mathbf{u} \cdot \mathbf{u}} \\
\dot{\theta}=\sqrt{\frac{\mathbf{a}_{\mathbf{p}} \cdot \mathbf{a}_{\mathbf{p}}}{\mathbf{u} \cdot \mathbf{u}}} ; \mathbf{e}_{\mathbf{f}}=\frac{\mathbf{u} \times \mathbf{a}_{\mathbf{p}}}{\left\|\mathbf{u} \times \mathbf{a}_{\mathbf{p}}\right\|}
\end{gathered}
$$


It should be noted that $\left(\mathbf{u} /\|\mathbf{u}\|, \mathbf{a}_{\mathbf{p}} /\left\|\mathbf{a}_{\mathbf{p}}\right\|, \mathbf{e}_{\mathbf{f}}\right)$ defines a Frenet frame attached to the fluid element, e.g. [31]. Also, $\dot{\theta}$ is related to the trajectory curvature $\kappa$, which has been the focus of recent studies in turbulent flows [31, 32], $\dot{\theta}^{2}=\kappa^{2} \mathbf{u} \cdot \mathbf{u}$.

The lamination rate of a material line element $\mathbf{d} \ell$ around the direction $\mathbf{e}_{\mathbf{f}}$ is defined using the spatial variation of $\dot{\theta}$. This spatial variation is evaluated by $\nabla \dot{\theta}$ and represents the differential winding or twisting as introduced by [2] for the Linked Twist Map. For each element $\mathbf{d} \ell$ of a material line $\ell$, a folding rate tensor is built based on the local gradient of the Lagrangian angular velocity. The product of a material line element by this tensor give the lamination rate around the directions $\mathbf{e}_{\mathbf{f}}$, for each element $\mathbf{d} \ell,[33]$ :

$$
\dot{R}_{f o l}=\int_{\ell}|\nabla \dot{\theta} \cdot d \ell|
$$

To extract the lamination rate potential of flows for a given length-scale $\mathcal{L}$, the folding rate intensity is simplified to

$$
\dot{R}_{f o l}=\|\nabla \dot{\theta}\| \mathcal{L}
$$

where $\nabla \dot{\theta}$ has been filtered at size $\mathcal{L}$. This formulation considers that the material line $\mathcal{L}$ is perfectly aligned with the gradient of rotation to highlight the laminating potential of flows without computing all possible shapes taken by $\mathcal{L}$. In practice, to consider the direction of the lamination rate, the value of $\nabla \dot{\theta}\left(\mathbf{e}_{\mathbf{f}} \cdot \mathbf{e}_{\mathbf{c}}\right)$ is filtered by averaging within mobile windows of size $\mathcal{L}$. $\mathbf{e}_{\mathbf{c}}$ represents either an axis of reference or a curvilinear reference. For example, this curvilinear reference can be chosen as the center-line of a vortical structure.

This quantification applies to whorls, tendrils and any flow structure. It is the persistence and coherence in time and in space that indicates the type of laminated structures generated. When the spatial variation of the Lagrangian angular velocity is coherent and sustained over a given domain, the striations within this domain increase with its size. The lamination rate potential increases similarly with the length-scale over coherent domains.

\subsection{Measure of lamination}

The lamination is measured by estimating the total length of the material line within circles (sphere in 3D) of diameter $\phi$, centred on any point of the material line, and then dividing it by $\phi$ :

$$
\mathcal{M}_{l}=\frac{1}{\phi} \int_{l \in d i s c} d l
$$

The position of the circle gives the spatial position of the measure and the diameter of the circle corresponds to the length-scale considered. It should be noticed that when $\phi \rightarrow 0$ then $\mathcal{M}_{l} \rightarrow 1$. For values of $\int_{l \in \text { disc }} d l$ larger than $\phi$, the line is considered to be folded and/or striated. Hence, the higher the value of $\mathcal{M}_{l}$, the higher the likelihood that the line pattern is laminated. If the lamination is homogeneous, $\mathcal{M}_{l}$ should increase with $\phi$. If the lamination is local, i.e. takes place within a flow structure of characteristic length-scale $\phi_{c}$, then $\mathcal{M}_{l}$ should decrease locally when $\phi$ increases and $\phi>>\phi_{c}$.

Maps of lamination indicate the location and the intensity of the laminated regions. They are built by considering a large number of material lines and extracting 
the lamination along these lines.

The circular measure is performed along material lines initially straight which are tracked in time. Fig. 5 shows a line after a time $t$, deformed by a velocity field, and two circles of same diameter for two different positions along the material line. For the circle on the left, the line appears as straight and the lamination measure $\mathcal{M}_{l}$ will be close to 1 . For the circle on the right, three line segments are crossing the circle: the central one is slightly bent, the segment above it is straight, and the segment beneath it is folded. These three segments contribute to the lamination of the material line within the domain defined by the circle. For this case, the lamination coefficient $\mathcal{M}_{l}$ of this line will be between 2 and 3 . The present measure goes beyond the quantification of curvatures and single bending. Indeed, this measure can estimate the lamination related to bending, rolling and the accumulation of lines within the measurement domain.

\section{Electromagnetically driven flows}

Electromagnetic forces, $\mathbf{f}(\mathbf{x})$, with $\mathbf{f}=\mathbf{j} \times \mathbf{B}$, allow the generation of rotational body forces within flows, $[34,35]$. Both magnetic field $\mathbf{B}(\mathbf{x})$ and electric current density $\mathbf{j}(\mathbf{x})$ can be tuned to produce tailor-made actuations. This provide a great flexibility in term of design and adjustment of the forcing intensity. They have been used in a variety of fundamental and applied research in fluid mechanics, e.g. generation of chaotic and turbulent flows to study mixing [36, 37], generation of vortices [38, 39], drag reduction [40-43], flow separation delay [44], boundary layers manipulation [45], heat transfers enhancement [46, 47] and turbulent-like flows e.g. $[5,26]$. At low Reynolds number, such body forcing can also be used to design bespoke flows, [17].

Fig. 6 shows a schematic of the shallow layer flow driven by electromagnetic forcing used in the present study. The magnetic field is produced by permanent magnets placed underneath the brine supporting wall. An electrolysis, imposed by positive and negative electrodes placed on both side of the tank, control the electrical current crossing the brine. The brine is made by mixing $158 \mathrm{~g} / \mathrm{l}$ of salt $(\mathrm{NaCl})$ with tap water. The viscosity $\nu$ is $1.3310^{-6} \mathrm{~m}^{2} / \mathrm{s}$, the density $\rho$ is $1105 \mathrm{~kg}$ $m^{-3}$ and the conductivity $\sigma$ is $16.6 \mathrm{~S} \mathrm{~m}^{-1}$, [48].

In the present case, the fluid conductivity $\sigma$ is low and the magnetic field is stationary. The induction equation can then be simplified to a purely diffusive magnetic field, $\nabla^{2} \mathbf{B}=0$. In addition, the relative velocity between the flow and the permanent magnet is low and the Ohm's law can be simplified to $\mathbf{j}=\sigma \mathbf{E}$ where $\mathbf{E}$ is the imposed electric field. The electromagnetic forces are consequently assumed to be independent of the flow velocity (for more details see $[34,45,49,50]$ ).

\subsection{Experiments}

Experiments are performed in a tank of horizontal size, $500 \mathrm{~mm} \times 800 \mathrm{~mm}$, with a brine supporting wall of size, $500 \mathrm{~mm} \times 600 \mathrm{~mm}$. The thickness of the brine is $H=6 \mathrm{~mm}$. The brine-supporting walls are kept straight and horizontal, with a standard deviation of the order of $0.1 \mathrm{~mm}$. A single-pair of cubic NdFeB magnets is supported by a $2 \mathrm{~cm}$ thick iron plate. The side-length of the magnets is equal to $L_{M}=40 \mathrm{~mm}$ and they are spaced by $40 \mathrm{~mm}$, see top view given in Fig. 7a. Their magnetic intensity is $B_{r}=0.68 \mathrm{~T}$. The North and South top surfaces are placed at $3.7 \mathrm{~mm}$ from the brine. The combination of the magnetic field generated by this pair of permanent magnets is combined with a quasi-uniform electric current 
density $\left(\mathrm{j}=13.5 \mathrm{~A} / \mathrm{m}^{2}\right.$ with $\left.\mathrm{I}=48.6 \mathrm{~mA}\right)$ to produce electromagnetic forces. The geometry of the forcing is then controlled by the position of the magnets which is given by the angle between the pair of magnets and the electric current, Fig. 7(a).

The raw velocity field is measured by Particle Image Velocimetry (PIV) using a $14 \mathrm{~Hz}$ camera $(2048$ pixels $\times 2048$ pixels, 14 bit $)$ and a picture frame of $441 \mathrm{~mm} \times 441 \mathrm{~mm}$. Pliolite DF01 particles (diameter varying between $100 \mu \mathrm{m}$ and $200 \mu \mathrm{m}$, density: 1.03) are used to seed the free surface. PIV post-processing is performed with an in-house code (iterative method with sub-pixel accuracy for the measured displacements). The typical maximal displacements are about 16 pixels per frame and the smallest correlation window of the iterative process is of 16 pixels $\times 16$ pixels. The characteristic values of the correlation coefficients of these measurements are about 0.9 . The PIV grids are computed with $222 \times 222$ points leading to a distance between two velocity points of 8 pixels. The experimental data are well resolved in space and time: the length of the PIV correlation window is more than ten times smaller than the magnets length with, an overlap of $50 \%$ and the temporal resolution allows real-time PIV between consecutive pictures for a continuous sequence. This enables the extraction of the spatial distribution of the velocity and acceleration fields via an hybrid PIV-PTVA (Particule Tracking Velocimetry and Accelerometry) procedure. The PTVA algorithm [51] is applied on a virtual Lagrangian tracking (the temporal integration is performed using 4th order Runge-Kutta algorithm, with a time step adjusted to a quarter of the mesh size divided by the maximum velocity, and the spatial interpolation is performed using a 2nd-order Lagrange polynomial). Note that real-time tracking based on the PIV data has been tested, but no improvement was gained compared to PTVA based on virtual tracking. It mainly added non-negligible noise when compared to virtual tracking. A very large number of virtual particles are tracked. This is to ensure that more than 18 positions are present in almost each window of 16 pixels $\times 16$ pixels. New grids are then extracted, while keeping a distance of 8 pixels between two grid points. The PTVA algorithm [51] is run with a $\Delta_{\text {target }}=32$ pixels which corresponds to twice the size of the smallest correlation windows. This ensures that eddies as small as 5 pixels in diameter (i.e. smaller than the mesh size) can be resolved by the PTVA algorithm. We favor this approach rather than a direct derivation of the space and time resolved PIV data, as this improve the quality.

Experiments are performed with a constant electromagnetic forcing. The reference scale for acceleration is $a_{r e f}=\frac{1}{\rho} f_{r m s} \simeq 0.003 \mathrm{~m} / \mathrm{s}^{2}$, where $f_{r m s}$ is the root mean square of the electromagnetic forces over the measurement domain. Different flow geometries can be generated by varying the orientation of the magnets with respect to the electric field. This induces variations of root mean square values (over the measurement domain) of velocity, $u_{r m s}$, and Lagrangian acceleration, $a_{r m s}$. Table 1 gives the corresponding values for the three orientations considered: $0^{\circ}, 15^{\circ}, 90^{\circ}$.

In the following, distances are non-dimensionalised using the magnet size $L_{M}$, the reference velocity is $u_{r e f}=\sqrt{\frac{f_{r m s} L_{M}}{2 \rho}} \simeq 0.015 \mathrm{~m} / \mathrm{s}$, and the reference lamination rate is $\dot{R}_{o l-r e f}=a_{r e f} / u_{r e f} \simeq 0.19 \mathrm{rad} / \mathrm{s}$. The reference time is simply defined as the ratio between the reference length and velocity. Non-dimensional quantities are indiced with the superscript ${ }^{+}$, i.e. $\dot{R}_{\text {fol-rms }}^{+}=\dot{R}_{f o l-r m s} / \dot{R}_{f o l-r e f}, \mathcal{L}^{+}=\mathcal{L} / L_{M}$, $t^{+}=t / t_{r e f}$. 


\begin{tabular}{ccccc}
\hline & & & \\
Angle & $u_{r m s}$ & $a_{r m s}$ & $a_{r m s} / u_{r m s}$ \\
\hline $0^{\circ}$ & 1.73 & 0.291 & 5.95 \\
$15^{\circ}$ & 1.88 & 0.297 & 6.32 \\
$90^{\circ}$ & 1.98 & 0.265 & 7.47 \\
\hline
\end{tabular}

Table 1. Main flow characteristics. $u_{r m s}$ is in $\mathrm{mm} / \mathrm{s}, a_{r m s}$ is in $\mathrm{mm} / \mathrm{s}^{2}, a_{r m s} / u_{r m s}$ is in s${ }^{-1}$.

\begin{tabular}{cccccc}
\hline Size $(\mathrm{cm})$ & 4 & 8 & 16 & 32 & 64 \\
\hline$j_{x}$ & 13.3 & 12.46 & 12.03 & 11.803 & 11.69 \\
$a_{r m s} 0$ & 0.0147 & 0.0115 & 0.00756 & 0.00433 & 0.0023 \\
$a_{r m s} 15$ & 0.0136 & 0.0107 & 0.0072 & 0.0042 & 0.00228 \\
$a_{r m s} 90$ & 0.0147 & 0.0116 & 0.00816 & 0.00492 & 0.00267 \\
$u_{r m s} 0$ & 0.129 & 0.146 & 0.156 & 0.161 & 0.163 \\
$u_{r m s} 15$ & 0.131 & 0.147 & 0.158 & 0.162 & 0.164 \\
$u_{r m s} 90$ & 0.155 & 0.171 & 0.182 & 0.188 & 0.19 \\
\hline
\end{tabular}

Table 2. Values used for simulations, for the three configurations $\left(0^{\circ}, 15^{\circ}\right.$ and $\left.90^{\circ}\right) \cdot j_{x}$ is in $A / m^{2}, u_{r m s}$ and $a_{r m s}$ are non-dimensionalized using $\frac{1}{\nu \rho} f_{r m s} H^{2}$ and $\frac{1}{\rho} f_{r m s}$, respectively.

\subsection{Numerical simulations}

All direct numerical simulations presented are carried out with a finite difference code, using 6th-order compact scheme for spatial discretization and 3rd order Runge-Kutta scheme for time derivatives. Poisson equation is solved directly using Fourier decomposition. The electromagnetic forcing model is based on an analytical model $[35,52]$, considering the 3D distribution of the permanent magnets. Previous numerical simulations have been carried out and validated for different intensity of the forcing, geometries and brine thickness which were chosen accordingly to experiments (more details in $[17,50]$ ).

Magnets are modeled with a height equal to $2 L_{M}$ and a magnetic intensity at the center of $B_{r}=0.68 \mathrm{~T}$. The brine thickness is constant and set to $H=5 \mathrm{~mm}$, its density $\rho=1105 \mathrm{~kg} / \mathrm{m}^{3}$, its kinematic viscosity $\nu=1.3310^{-6} \mathrm{~m}^{2} / \mathrm{s}$.

In section 4.2 .2 , the size of the magnets varies from $L_{M}$ to $16 L_{M}$, and the distance between the brine of the upper surface of the magnets is set equal to the tenth of the magnet size. $f_{r m s}$ is kept constant by adjusting the electrical current density. The simulations are performed with a minimal resolution $\left(n_{x}, n_{y}, n_{z}\right)$ of $129 \times 17 \times 129$ within a domain of size $10 L_{M} \times H \times 10 L_{M}$ and with wall (no-slip) boundary conditions. Results are extracted at $y=0.625 \mathrm{H}$.

\section{Mono-scale forcing}

Three canonical flow structures, driven by electromagnetic forcing, are used to assess the definition of lamination and lamination rate (Secs. 2.1 and 2.2). A number of experimental and numerical studies have been performed for a large range of forcing scales and for different orientation of the magnets. 


\subsection{Flow structures}

Different flow geometries can be generated by the pumping of the flow above a pair of permanent magnets, Fig. 8(a), depending on the orientation of the magnets, with respect to the electrical current. The flow configurations are named according to this angle. For all cases, the flow is laminar, quasi-two-dimensional and quasisteady for the low forcing intensity considered [17].

The configuration $0^{\circ}$ corresponds to the impact of two opposite jets. These jets produce a region of high strain around an hyperbolic stagnation point. With orthogonal manifolds, this region can locally be considered as pure strain. In addition, this configuration also produces four smaller recirculating regions.

As shown in Fig. 8(c), these recirculating regions correspond to domains where the Lagrangian acceleration is converging toward focus points which are located at the centre of the recirculating domain. The high strain region produced by the impacting jets corresponds to a domain where acceleration lines are emanating from a source point.

The configuration $15^{\circ}$ corresponds to the impact of two jets which are slightly off-axis. This produces a region of high strain with non-orthogonal manifolds, i.e. a combination of pure strain and shear. Four recirculating regions are also produced. In this case, two recirculating regions are smaller and of stronger velocity intensity than the other two. The streamlines around these two recirculating regions create a "cat's eyes" shape.

The configuration $90^{\circ}$ corresponds to a single recirculation, eddy-like structure, generated by two parallel jets in opposite directions. The topology and geometry for this flow is different from the previous two configurations, both for velocity and acceleration. Still, the recirculation corresponds to acceleration lines converging toward the focus node of the recirculation region.

Visualisations of an initially regular grid are shown on Fig. 9, for all three configurations. Grids are tracked during many turnover times and their temporal evolution can be seen on videos movgrid0.avi, movgrid15.avi, movgrid90.avi. Grids deformation of Fig. 9 are extracted after one turn-over time, based on the magnets length and $u_{r m s}$. For all cases, the grid is mostly deformed in the central part of the domain, where the velocity and velocity gradient are high. For $0^{\circ}$, a strong deformation in regions of high strain, close the hyperbolic stagnation point, is observed. Fig. 9, $0^{\circ}$, also shows a rolling of the lines within the recirculating domain. At $15^{\circ}$, the grid visualisation reveals the "cat's eyes" shape, with a strong rolling of the line around both "eyes". Bent and folded lines can also be observed above the magnets, where the flow is pumped, and in the region emanating from the hyperbolic domain associated with the central stagnation point. The visualisation for $90^{\circ}$ shows a rolling of the grid lines around the center of the recirculation zone. The grid is only slightly deformed within the central region, for what look like a solid body rotation.

\subsection{Identification of the laminating rate}

Visualisations of Fig. 9 and corresponding animations show that the three flows considered present different laminating patterns. The quantification of laminating rate presented in section 2.1 is now applied to these configurations, in order to extract the mechanisms leading to lamination.

\subsubsection{Scales and patterns of the lamination rate}

To characterize the lamination rate, Eq. 5 is computed over the whole domain, using the procedure described in section 2.1. For practical consideration, the small- 
est length-scale accessible is equal to the size of the PIV mesh $\ell_{m}$.

First the rms of the lamination rates, $\dot{R}_{\text {fol-rms }}^{+}$, is estimated for scales varying from the mesh size to four times the size of the magnet, i.e. from $\mathcal{L}^{+}=0.043$ to $\mathcal{L}^{+}=4$. Fig. 10 gives the evolution of $\dot{R}_{\text {fol-rms }}^{+}$as a function of the non-dimensional length $\mathcal{L}^{+} . \dot{R}_{\text {fol-rms }}^{+}$reaches its maximum values for typical length-scale about one decade higher than the mesh size, and for a scale just above the magnet size. This indicates that these flow structures laminate more efficiently at a particular length-scale. For the configuration $15^{\circ}$, this length-scale is slightly higher, and the maximum is found at $\mathcal{L}^{+}=1.38$. This value is noted $\mathcal{L}_{\text {fol }}^{+}$, and will be used later as a reference scale. Also, it is striking that the configuration $90^{\circ}$ has a much lower lamination rate than the two other configurations for length smaller than $\mathcal{L}^{+}=2$, which corresponds to the spacing between the magnet centres. Compared to $0^{\circ}$ and $15^{\circ}$, the lamination rate is becoming significant at $90^{\circ}$ only for length-scales larger than $\mathcal{L}^{+}=1$. This is in good agreement with the visualisations given in Fig. 9 where the main lamination occurs around the center of the recirculation zone.

The spatial distribution of $\dot{R}_{\text {fol }}^{+}$are given in Fig. 11 for $\mathcal{L}=\ell_{m}, \mathcal{L}=\mathcal{L}_{\text {fol }} / 2$ and $\mathcal{L}=\mathcal{L}_{\text {fol }}$. For all three cases, the lamination rate magnitude is minimum at the mesh size. In particular at $90^{\circ}$, it is one decade lower than at $\mathcal{L}_{\text {fol }}^{+}$, and not much above the noise level of the measure. For $0^{\circ}$ and $15^{\circ}$, the lamination rate observed at the mesh size is concentrated in the region surrounding the hyperbolic stagnation point. Close to this point, the acceleration redistributes the velocity in a very sharp way. This abrupt change in direction is illustrated by the streamlines in Fig. 8.

At large scale $\left(\mathcal{L}=\mathcal{L}_{\text {fol }} / 2\right.$ and $\left.\mathcal{L}=\mathcal{L}_{\text {fol }}\right)$, the lamination rate intensity is higher over larger portion of the domain, for all flow configurations. The largest intensities are still found along the principal directions. These directions correspond to the direction of V-shaped bands observed in the visualisations, Fig. 9, and to the stable and unstable manifolds associated with the central hyperbolic stagnation points in cases $0^{\circ}$ and $15^{\circ}$. In addition, local minima of lamination rate intensity are associated with regions of weaker grid deformation. A comparison between Fig. 8 and Fig. 9 shows that these regions correspond to the center of the recirculations.

\subsubsection{Dependence of lamination rate with the flow structures length-scale}

The dependance of lamination rate with the length-scales is now explored. As the lamination rate is expected to be controlled by the forcing length-scale, the size of the magnets $L_{M}$ is varied from $4 \mathrm{~cm}$ to $64 \mathrm{~cm}$ using numerical simulations.

The electromagnetic forces and the brine thickness being kept constant, the rms velocity $u_{r m s}$ increases with the magnet size, due to the extension of the acceleration domain above the magnets. It should be noted from table 2 that this increase is moderate. Indeed, it does not grow indefinitely with the extension of the forcing domain. A particle starting with a zero velocity and entering a domain forced uniformely will see its speed $u_{p}$ increasing in time. This temporal evolution can be simplified by considering a linear drag due to the bottom friction $-\alpha \mathbf{u}$ with $\alpha \simeq \frac{\nu}{H^{2}}:$

$$
\frac{\partial u_{p}}{\partial t}=\mathbf{f}-\alpha \mathbf{u} ; u_{p} \sim \frac{f}{\alpha}\left(1-e^{-\alpha t}\right)
$$

where $t$ is the crossing time of the particle in the force field. Due to the bottom friction, the rms velocity is bounded when increasing the size of the forcing domain whilst keeping the intensity of the forces constant.

There is also a significant evolution of the rms of the Lagrangian acceleration 
which indicates that the spatial distribution of the Lagrangian acceleration is characteristic of the flow structure and length-scale. The driving forces and brine thickness being kept constant whilst the velocity magnitude does not vary significantly, it is the pressure distribution (depending on the magnets spacing controlling the structure size) which drives this variations of Lagrangian acceleration, thus confirming the key role of pressure observed in [50].

Importantly, the characteristic frequency of the flow, given by the ratio $a_{r m s} / u_{r m s}$, varies for more than one decade with the increase of the magnet size (Fig. 12). In addition, it is found that $\frac{a_{r m s}}{u_{r m s}} \sim\left(\frac{L_{M}}{H}\right)^{-3 / 4}$.

Figure 13 gives the evolution of the rms of the lamination rate, $\dot{R}_{f o l-r m s}$, nondimensionalized using $a_{r m s} / u_{r m s}$ as a function of the length-scale, scaled by the different magnets sizes and for the three forcing configurations: $0^{\circ}, 15^{\circ}$ and $90^{\circ}$. For all configurations, the curves collapse to similar magnitudes, showing that the lamination rate intensity scales with $a_{r m s} / u_{r m s}$, and consequently:

$$
\dot{R}_{f o l} \sim \dot{R}_{f o l_{0}}\left(\frac{\mathcal{L}}{\mathcal{L}_{0}}\right)^{-\frac{3}{4}}
$$

where $\mathcal{L}$ is the flow structure length-scale and the zero index indicates chosen references. These results support the fact that the lamination rate scales with the flow structure and that the lamination rate increases when flow structures lengthscales are reduced.

The two configurations based on the generation of impacting jets $\left(0^{\circ}\right.$ and $\left.15^{\circ}\right)$ show very similar curves. This indicates that the geometry of the forcing controls the flow structure, hence the lamination rate. For $90^{\circ}$ (Fig. 13c), the lamination rate depends more on the magnet size. In fact, when the size of the magnets increase, the turnover time, based on the rms velocity (over the centre part of the domain, $\left.5 L_{M} \times 5 L_{M}\right)$ and the magnet size $L_{M}$, tends to be much larger than the typical viscous time due to the bottom friction $H^{2} / \nu$. This last term is thus expected to play an increasing role and isolates the magnets as $L_{M} / H$ increases. This clearly modifies the spatial distribution of the lamination rate. The two velocity fields shown on Figure 14 confirm this assertion. For $L_{M}=4 \mathrm{~cm}$, the forcing generates an eddy-like recirculation, while for $L_{M}=64 \mathrm{~cm}$, the magnets are isolated from each other, resulting in a cat's eye flow structure of weak velocity intensity in the centre. This evolution explains the variation of the lamination rate profiles observed in Figure 13 when $L_{M} / H$ is increased.

\subsection{Potential lamination and comparison with grid deformation}

The lamination of a flow is an integrative process along the Lagrangian trajectories of the particles defining a material line. To stress the ability of the proposed lamination rate to quantify the laminating properties of flows, $\dot{R}_{f o l}^{+}$is averaged over a time $t$ along trajectories tracked backward in time, allowing the extraction of potential lamination. The distribution and the intensity of the potential lamination (at a given size, $\mathcal{L}$ ) are then quantified after short and long times. The long time of reference chosen is typically about one turn-over time. The short time-scale is $1 / 8$ of this turn-over time. The final test is performed by comparing visualisations, starting at $-t$ with a regular distribution of black bands, with these mean lamination quantities. If the measure of $\dot{R}_{\text {fol }}^{+}$is coherent, laminated domains (at size $\mathcal{L}$ ) should be found where the backward integration is the highest.

For the length-scale, $\mathcal{L}=\mathcal{L}_{\text {fol }}$, Fig 15 shows color maps obtained after a short 
$\left(t^{+}=0.61\right)$ and long $\left(t^{+}=4.9 \sim L_{M} / u_{r m s}\right)$ integration time. Please note that the scales are indicated by white bar on all plots. The deformed grids are superimposed on the integrated results. For the shortest integration time, and for all three cases, the grids are bent around maxima of lamination rate. This definition captures the bending properties of the local "jets" observed in Fig. 8. For longer integration time, and for the three configurations, there is a good correspondence between grid deformation and spatial distribution of the mean lamination rate. For $0^{\circ}$, the maximum lamination is observed where the mean of $\dot{R}_{f o l}^{+}$is maximum. The four circular areas, around the two main axis, have small integral values, and they correspond to region where the flow has been weakly folded, as shown by the persistence of the straight, almost perpendicular, lines. For $15^{\circ}$, the eight shape of the visualisation pattern superimposes well with the map. The local minima of the integration correspond to a weaker lamination of the visualitation pattern and the local maxima correspond to material lines which have been bent and/or rolled. Finally, at $90^{\circ}$, the central part of the flow appears to be in solid body rotation and the lamination is very weak in this region. The main folding is found further away from the center, around this recirculation.

For a smaller scale $\left(\mathcal{L}=\mathcal{L}_{\text {fol }} / 2\right)$, the superimposed grids and maps are shown on Fig. 16. Those results are globally similar to those at size $\mathcal{L}_{\text {fol }}$. Due to the smaller length-scale, the maps looks sharper than in Fig. 15. For a longer integration time, a few differences can be observed. At $0^{\circ}$, maxima occur closer to the main axis, where the strongest line folding, issuing from the central part of the domain, is observed. Also, the weakest lamination within the center of the four recirculation regions is less noticeable for this map. For the $15^{\circ}$ case, there is also a weaker lamination rate within the center of recirculation zone. The main difference comes from sharper details and from the reduction of the lamination potential intensity beside the eight shape. At $90^{\circ}$, both results at size $\mathcal{L}_{\text {fol }} / 2$ and $\mathcal{L}_{\text {fol }}$ are very similar, and only small details around the edges of the active region differ from one filtering scale to the other.

Profiles along selected axis indicated by white arrows on Figs. 15 and 16, are plotted in Fig. 17. For all three configurations, the lamination potential has welldefined local maxima, and varies significantly in intensities with the length scale. Except, for $90^{\circ}$, the local maxima are slightly higher for the lamination potential at size $\mathcal{L}_{\text {fol }} / 2$ than at size $\mathcal{L}_{\text {fol }}$. For $0^{\circ}$, the values are extracted along a vertical line crossing two recirculation zones and a region of strong folding. The lamination potential at size $\mathcal{L}_{\text {fol }} / 2$ shows a peak close to the unstable manifold of the central hyperbolic stagnation point, where the lines are folded in V-shape. At this size, secondary plateaux are observed above the two recirculating regions. For $\mathcal{L}_{\text {fol }}$, the central peak previously observed vanishes and merges with the lamination potential due to the rolling of the recirculating zones. For the $15^{\circ}$, lamination rate is taken along an horizontal line crossing one of the two strongest recirculation zones. The plateau observed between $x^{+}=3$ and $x^{+}=4$ for $\mathcal{L}_{\text {fol }}$ disappear when $\mathcal{L}^{+}$is reduce, because the bending of the black lines in this region is mainly observed at large scale. One interesting feature is the sharp drop which is observed between $x^{+}=4.5$ and $x^{+}=5$. This drop corresponds to the boundary between the recirculation zone and the region affected by the upper jet. The grid visualisation confirms that two different lamination patterns occurs on both side of this point. For $90^{\circ}$, the values are exported along an horizontal line crossing the centre of the recirculation. The two curves for $\mathcal{L}_{\text {fol }}$ and $\mathcal{L}_{\text {fol }} / 2$ are very similar, with values slightly higher at large scale than at small scales.

Both large and small-scale lamination potential are in agreement with the superimposed grid deformation. These results show the coherence of the proposed 
lamination rate. The agreement found for these three typical flows support the fact that lamination rate can now be quantified to explore lamination in more complex flows.

\subsection{Measure of lamination}

In order to measure lamination, material lines are now tracked in time for the three flows considered above. They are randomly generated from wall to wall. Each line is formed of particles which are tracked in time using a Runge Kutta scheme for the advancement in time. The distance between neighbouring points is kept smaller than $1 / 8$ of the PIV mesh size. If the distance between neighbouhring points is larger than this size, a particle is added within the initial (at $t=0$ ) distribution of the particles forming the material line. More than 48,000 lines are tracked to generate the statistics, with more than 250 million particles.

Even if the flow is quasi-two-dimensional and quasi-steady, the time evolution of these lines is not obvious. Fig. 18 shows some illustrations. There is an extremely rich variety of possible patterns, e.g. both lines for $15^{\circ}$ are very different. Also, the lines shown on Fig. 18 do not cover the entire family of line evolutions. In addition, the dependence of the lamination to the initial position and orientation of the material line is important. This justifies the large number of lines and particles tracked for each case. The two temporal animations for the lines corresponding to the $15^{\circ}$ case, can be found in movies mov-line-or15_A-mp4.avi and mov-lineor15_B-mp4.avi.

Fig. 19 gives the average (over all realisations) distribution of lamination measures (Section 2.2) for the three flow configurations. Three examples, corresponding to $\phi=L_{M} / 2, L_{M}$ and $1.5 L_{M}$ have been selected to illustrate the evolution of the lamination maps with the diameter of the circle.

For $0^{\circ}$ (first row, Fig. 19), the lamination measured increases with the size of the circles. This is due to the integration of lamination over an increasing number of scales. For a diameter $\phi$ smaller than $L_{M}$, lamination coefficients are lower than 2. Maxima are found where the flow is bent. For $\phi=L_{M}$, areas where the lamination accumulates due to the flow recirculation appears. When the size is increased further $\left(\phi=1.5 L_{M}\right)$, the importance of recirculation regions increases. An asymmetry, also noticeable for the lamination rate, can be observed in the bottom left quarter and is due to experimental conditions. Indeed, such asymmetry is difficult to see by just considering velocity and acceleration independently. For $15^{\circ}$, (second row, Fig. 19), the intensity of laminated domains increases significantly with the diameter of the circles. In particular, the cat's eyes structure is found to strongly laminate within (and around) each eye. Also, this local level of lamination is significantly higher for the $15^{\circ}$ than for the $0^{\circ}$ configuration. For $90^{\circ}$, (third row, Fig. 19), and for the three diameters, the lamination is always smaller than 2. Lamination domains are mainly found at the edge of the recirculation region and above the magnets.

\section{Application to multi-scale flows with turbulent-like properties}

The lamination characterization and lamination rate are now applied to laminar multi-scale flows with turbulent-like properties [5, 26]. 


\subsection{Flow structure}

Experimental and numerical results describing velocity and acceleration fields for the multi-scale arrangement of magnets can be found in [50]. The three forcing length-scales (i.e. size of magnets) are $0.25 L_{M}, L_{M}$ and $4 L_{M}$. The multi-scale generation of counter-jets creates a structure of "eddies-within-eddies". The topology of these flows are quite similar for the range of forcing considered. The energy spectra and pair separation statistics of these flows are similar for a broad range of Reynolds numbers [5, 26, 50]. However, the details appears to be quite different. Indeed, the visualisation given in Fig. 20 highlights a lamination within eddies at intermediate scales for $R e=3$ which is much more intense than for $R e=25$, with $R e=u_{r m s} H / \nu$.

\subsection{Lamination rate}

Fig. 21 gives the evolution of the root mean square (over space) of the lamination rate as a function of filtering length-scales. For low Reynolds numbers, a peak of lamination appears for a length-scale slightly larger than the medium scale of forcing. This local maximum of lamination rate is consistent with the lamination of material bands observed on Fig. 20, where laminated domains are observed within intermediate size recirculations. For higher Reynolds numbers, this peak disappears. At intermediate Reynolds numbers, a plateau of lamination rate can be observed between $\mathcal{L}^{+}$and $6 \mathcal{L}^{+}$.

The lamination rate maps are plotted in Fig. 22, for three different scales $\left(\mathcal{L}^{+}=0.4,1.6\right.$ and 6$)$ for $R e=3$ and $R e=25$. The plots at intermediate and large scale respectively correspond to the peak of lamination and to the end of the plateau on Fig. 21. The lamination rate at small length-scales, $\mathcal{L}^{+}=0.4$, is mainly observed above the small and medium magnets. When the length scale increases, the intensity of the lamination rate tends to decrease and the laminated region tends to increase. This indicates that the lamination rate are more intense at small-scales. Nevertheless, for the multi-scale flows considered, the small scales cover only a small part of the flow domain. Consequently, they have a small impact on the global statistics of Fig. 20.

There are also significant differences in spatial distribution of lamination rate between $R e=3$ and $R e=25$. The lamination rates are more intense at small and medium scales for $R e=3$ than for $R e=25$. Lamination is thus expected to be different at Reynolds $R e=3$ and $R e=25$, which is in agreement with Fig. 20.

These results show that the lamination rate is sensitive to geometrical changes within multi-scale flows of similar topology and forcing. If statistics such as twoparticle dispersion, e.g. [53], may not be very sensitive to lamination, the evolution of material lines is strongly affected by the laminating properties of flows. The difference between the low and high Reynolds numbers demonstrated for these flows shows the importance of the lamination rate to complement existing description of mixing and stirring properties within complex flows.

\subsection{Lamination potential and comparison with grids deformation}

Fig. 22 compares the potential lamination maps with the grid deformation given in Fig. 23. As for the mono-scale case, the agreement between grid deformation and lamination potential is good, particularly at small and medium scales. Indeed, bending and rolling of material bands are observed close to maximum potential.

For small length-scales, $\mathcal{L}^{+}=0.4$, (Fig.23a), the lamination potential is found 
with maxima above small flow structures. For $R e=3$, rings of higher intensities are also observed around the recirculation associated to the medium scales of forcing. For $R e=25$, these rings are replaced by tongues of lower intensities.

For intermediate length-scales, $\mathcal{L}^{+}=1.6$, (Fig.23b), a high lamination potential appears within the recirculations associated with the medium scale of forcing for the low Reynolds number case. Also, elongated regions of high lamination potential intensity are noticed for both Reynolds numbers.

Finally, for the largest scale considered here, $\mathcal{L}^{+}=6$ (Fig.23c), $R e=3$ has lower lamination potential values than at small scales. $R e=25$ shows a higher lamination potential for the flow in between scales of forcing. The agreement between lamination potential and grid deformation is less obvious at this scale as the material bands have been deformed at all scales and not only at $\mathcal{L}^{+}=6$.

\subsection{Measure of lamination within multi-scale flows}

Fig. 24 illustrates two different line tracking for $R e=3$ and $R e=25$. As for the mono-scale case, the possible patterns strongly vary with line positions and orientations. As expected, the multi-scale structure of the flow increases the complexity of the lines evolution.

Fig. 25 gives the distribution of the laminated domains using the measure, $\mathcal{M}_{l}$, of lamination within circles. Results are given for Reynolds numbers $R e=3$ and $R e=25$, and three circles diameters corresponding to the magnets sizes, $\mathcal{L}^{+}=0.25$, $\mathcal{L}^{+}=1$ and $\mathcal{L}^{+}=4$. For both Reynolds numbers, the maxima of lamination are dominated by the small scales. This is in agreement with shorter turn-over times above the small-magnets and also the discussion of section 4.2 .2 , where it was found that lamination rate increases with the reduction of the flow structures sizes. For both Reynolds numbers, the laminated domain increases with the diameter of the measurement circle.

For $R e=3$ and $\mathcal{L}^{+}=0.25$, laminated structures appear above the small recirculation regions, but they also appear in rings around the medium recirculation, and trails emanating from the medium-scale region. When the diameter of the circles is increased, $\mathcal{L}^{+}=1$, the lamination appears within medium-scale recirculation and is still strong at small-scales. For the circle of diameter $\mathcal{L}^{+}=4$, the maxima are still found at small and medium-scales. Domains of lamination also appear in between the previous structures due to the extension of the circle diameter.

For $R e=25$ and $\mathcal{L}^{+}=0.25$, the laminated structures mainly appear above the small scales. This pattern is conserved for circles of diameters $\mathcal{L}^{+}=1$. When the circle diameter increases to $\mathcal{L}^{+}=4$, the lamination measured above the small-scales reduces more than for $R e=3$. These reductions (for both $R e=3$ and $R e=25$ ) are due to the increase of the measurement domain whilst the highest lamination is local and at smaller scales than the considered diameter.

These results within multi-scale flows complement those obtained with singlescale forcing. They show that the measure of lamination can be an effective way to quantify domains which have been folded/striated and with what intensity they have been laminated.

\section{Relations between lamination rate and measure}

To the authors' knowledge, there is no exact relation between the lamination rate (Eq. 5) and the measure $\mathcal{M}_{l}$. This section is aimed at bridging both defintions. The relative growth $\mathcal{G}$ of a material line due to the lamination rate at size $\mathcal{L}$ and 
during time $T$ can be estimated as:

$$
\mathcal{G}(\mathcal{L}, T)=\int_{0}^{\mathcal{L}} \int_{0}^{T} \frac{\dot{R}_{f o l}(l, t)}{2 \pi l} d l d t
$$

It was shown in previous sections that the lamination rate is correlated with the measure of lamination. Considering that the growth given by equation 9 corresponds to an increase of the total length within circles of diameter $\mathcal{L}$, an expected relation should be in the form:

$$
\mathcal{M}_{l}(\mathcal{L}, T) \simeq 1+\alpha \mathcal{G}
$$

where $\alpha$ is a function of time- and length-scales considered, and could also depend on the flow.

Fig. 26 gives the evolution of the rms lamination measured and $\mathcal{G}$, for the three mono-scale and two multi-scale configurations. The temporal integration of the lamination potential is performed over the duration corresponding to the lamination measure, (Fig. 19 and Fig. 25). These times are $t^{+}=4.9$ for the mono-scale forcing cases and one-turnover time $\left(4 L_{M} / u_{r m s}\right)$ for the multi-scale cases.

For all the configurations, similar tendencies are observed. The same order of magnitude is found between the increase of the measure with $\mathcal{L}$ and the values of the integral of the lamination rate. These estimations rely on spatial and temporal integration over long durations of lamination rates varying by more than one decade in magnitude. Also, for both estimations, the configuration $15^{\circ}$ and $0^{\circ}$ are found to laminate more than the configuration $90^{\circ}$. Similarly, the multi-scale case $R e=3$ is found to laminate more than the case $R e=25$.

$\alpha$ is not far from a constant for the multi-scale flows, but it is not the case for the one-scale configurations. For the multi-scale configurations, $\alpha$ is close to 1.5 for both Reynolds numbers. Significant variation of $\alpha$ are observed for the mono-scale configurations for length-scales $\mathcal{L}^{+}$smaller than 1 . When the $\mathcal{M}_{l}$ is high enough (i.e. $>1.2$ ), $\alpha$ still tends to decrease with $\mathcal{L}^{+}$and $\alpha \sim 1.4$.

These results show a good agreement on the lamination intensity between the measure and the lamination rate approach, even if the relation between both quantities is not exact. In fact, it should be noticed that the measure via the estimation of the line length within circles integrate all laminating mechanisms from the smallest to the largest scales. The measure of lamination goes beyond the integration of the lamination rate proposed from the smallest to the length-scale considered. For example, the movie mov-line-or15_B-mp4.avi shows how two parts of the line, initially far away, are gathered together, increasing locally the lamination. Also, the proposed quantification of lamination rate relies on the alignment between Lagrangian acceleration and velocity, bending mechanisms related to maximum of velocity without variation of acceleration (e.g. 1D infinite Poiseuille flow) will not be captured by the proposed quantification of lamination rate whilst they will be by the measure $\mathcal{M}_{l}$.

The spatial comparison between the measure of lamination (Figs. 25 and 19) and the potential lamination at a given scale (obtained via backward integration, Figs. 23, 15 and 16) is not straightforward. Nevertheless, results are coherent for monoand multi-scale configurations. Considering the multi-scale forcing, this coherence includes the evolution of the lamination distribution with the length-scale. For example, in the case $R e=3$, the length-scale corresponding to the small magnets shows lamination rates leading to a higher lamination above the small scale of forcing, within a ring around medium-scale recirculations and within tails. A similar 
distribution is observed for the measure of lamination at the small-scale. Also, the measure of lamination at intermediate scales is well explained by the "addition" of lamination rates at small and medium scales of forcing, as lamination within the medium scale recirculations appears in the measure at this scale. In addition, the local values of lamination intensities compare well. The lamination increment measured within main recirculating regions is about twice as large for $15^{\circ}$ than for $0^{\circ}$, Fig. 19. A similar ratio is found between $15^{\circ}$ and $0^{\circ}$ for the potential lamination maps and curves, Fig. 25 and 17.

\section{Conclusion}

A quantification of lamination rate and a measure of lamination have been defined and applied to canonical (quasi-two dimensional and quasi-steady) one- and multiscale flows. The results presented in this paper support the use of the proposed quantification of lamination rate to explore lamination driving terms. They also support the use of the proposed measure of lamination to obtain an independent and complementary quantification of lamination.

An agreement is found between lamination potential and grid deformation. Similar agreement is found between lamination potential and the measure of lamination. Results are coherent for both intensity and spatial distribution. This shows that the quantification lamination rates proposed captures leading mechanisms to laminate and fold flows.

In addition, results discussed have highlighted the rich complexity of lamination and lamination rate, which are closely related to flow geometries. Lamination rate varies with the flow structure and various characteristics structures have been discussed. Both quantification of lamination rate and measure of lamination allow the extraction of the different stirring properties for the investigated flows. For example, the study of lamination within multi-scale flows reveals significant stirring differences whilst the energy spectra and topology are conserved for this family of flows.

One important results is the growth of the lamination rate and the lamination measured with the diminution of flow structures' length-scale which is observed for both one-scale and multi-scale configurations. In the context of turbulent flows, the growth of lamination at small scales could trigger baker-like processes and/or enhance locally mixing and diffusion by applying strain over striated scalar patterns. Also, in principle, this enhancement of diffusion due to the application of strain over striated scalar domains can occur at many scales, including scales smaller than Kolmogorov and Batchelor length-scales $[54,55]$. To date, such "multi-cycle" process is not considered in standard approaches of turbulent mixing and is still to be measured/studied in turbulent flows.

Lamination rate and measure should be two key elements for the design of new mixers. They should also allow a more complete description of mixing in complex and turbulent flows.

\section{acknowledgment:}

The authors acknowledge: The Royal Society, the EPSRC, Dr. G. Fishpool, S. Bocquet, J.M. Garcia de la Cruz. EPSRC Grant EP/D072034/1.

\section{References}

[1] O. Reynolds, "Study of Fluid Motion by means of coloured Bands", Proceedings of the Royal Institution of Great Britain XIV (1893), pp. 129-138. 
[2] S. Wiggins, and J.M. Ottino, "Foundations of chaotic mixing", Phil. Trans. R. Soc. Lond. A 362 (2004), pp. 937-970.

[3] G.A. Voth, G. Haller, and J.P. Gollub, Experimental Measurements of Stretching Fields in Fluid Mixing, Phys. Rev. Lett. 88 (2002), p. 254501.

[4] J.M. Ottino, "The kinematics of mixing", Cambridge University Press (1989).

[5] L. Rossi, J.C. Vassilicos, and Y. Hardalupas, Multiscale Laminar Flows with Turbulentlike Properties, Phys. Rev. Lett. 97 (2006), p. 144501.

[6] G. Haller, "Finding finite-time invariant manifolds in two-dimensional velocity fields", Chaos 10 (2000), pp. 99-108.

[7] B. Eckhardt, and D. Yao, Local Lyapunov exponents in chaotic systems, Physica D 65 (1993), pp. $100-108$.

[8] G. Haller, and G. Yuan, "Lagrangian coherent structures and mixing in two-dimensional turbulence", Physica D 147 (2000), pp. 352-370.

[9] K. Bajer, A.P. Bassom, and A.D. Gilbert, Accelerated diffusion in the centre of a vortex, J. .Fluid Mech. 437 (2001), pp. 395-411.

[10] J. Weiss, "The dynamics of enstrophy transfer in two-dimensional hydrodynamics", Physica D 48 (1981), pp. 273-294.

[11] A. Okubo, "Horizontal dispersion of floatable trajectories in the vicinity of velocity singularities such as convergencies", Deep-Sea Res 17 (1970), pp. 445-454.

[12] M. Chong, and A.P.B. Cantwell, A general classification of three-dimensional flow fields, Phys. Fluids A 2 (1990), pp. $765-777$.

[13] A.D. Stroock, S.K.W. Dertinger, A. Ajdari, I. Mezic, H.A. Stone, and G.M. Whitesides, Chaotic Mixer for Microchannels, Science 295 (2002), pp. 647-651.

[14] C. Simonnet, and A. Groisman, Chaotic Mixing in a Steady Flow in a Microchannel, Physical Review Letters 94 (2005), p. 134501.

[15] T. Cubaud, and T.G. Mason, Folding of Viscous Threads in Diverging Microchannels, Physical Review Letters 96 (2006), p. 114501.

[16] J. Frederick R. Phelan, N.R. Hughes, and J.A. Pathak, Chaotic mixing in microfluidic devices driven by oscillatory cross flow, Phys. Fluids 20 (2008), p. 023101.

[17] L. Rossi, S. Bocquet, S. Ferrari, J.G. Cruzde la, and S. Lardeau, "Control of flow geometry using electromagnetic body forcing", Int J. Heat Fluid Flow 30 (2009), pp. 505-513.

[18] R. Antonia, A. Chambers, D. Britz, and B. L.W., Organized structures in a turbulent plane jet: topology and contribution to momentum transport, J. Fluid Mech. 172 (1986), pp. 211-229.

$19]$ Z. Warhaft, passive scalars in turbulent flows, Annu. Rev. Fluid Mech. 200032 (2000), pp. 203-240.

[20] S. Goto, and J.C. Vassilicos, "Particle pair diffusion and persistent streamline topology in twodimensional turbulence", New J. Phys. 6 (2004), pp. 1-35.

21] Y. Dubief, and F. Delcayre, On coherent-vortex identification in turbulence, J. Fluid Mech. 553 (2000), pp. 227-252.

[22] K. Schneider, M. Farge, and Z.J. azzalini A., Coherent vortex extraction and simulation of $2 D$ isotropic turbulence, Journal of Turbulence 7 (2006).

[23] S. Robinson, "Coherent motions in the turbulent boundary layer", Annu. Rev. Fluid Mech. 23 (1991), pp. 609-39.

[24] R. Adrian, C. Meinhart, and C. Tomkins, Vortex organisation in the outer region of the turbulent boundary layer, J. Fluid Mech. 422 (2000), pp. 1-54.

[25] E. Hascoet, L. Rossi, and J. Vassilicos, Multi-scale flow control for efficient mixing: simulation of electromagnetically forced turbulent-like laminar flows, IUTAM Symposium on Flow Control and MEMS (2008), pp. 273-277.

[26] L. Rossi, J.C. Vassilicos, and Y. Hardalupas, Electromagnetically controlled multi-scale flows, J. Fluid. Mech. 558 (2006), pp. 207-242.

[27] L.F. Richardson, Proc. R. Soc. Lond. A 110 (1926), pp. 709-737.

[28] J. Thiffeault, Stretching and curvature of material lines in chaotic flows, Physica D 198 (2004), pp. $169-181$.

[29] J.M. Ottino, S.C. Jana, and V.S. Chakravarthy, From Reynolds's stretching and folding to mixing studies using horseshoe maps, Phys. Fluids 6 (1994), pp. 685-699.

[30] P. Tabeling, M. Chabert, A. Dodge, C. Jullien, and F. Okkels, Chaotic Mixing in Cross-Channel Micromixers, Philosophical Transactions: Mathematical, Physical and Engineering Sciences 362 (2004), pp. $987-1000$

[31] W. Braun, F.D. lillo, and B. Eckhardt, Geometry of particle paths in turbulence, Journal of Turbulence 7 (2006), pp. $1-11$

[32] H. Xu, T. Ouellette, and B. E., "Curvature of Lagrangian trajectories in turbulence", Phys. Rev. Lett. 98 (2007), p. 050201.

[33] L. Rossi, A mechanism to explore lamination rate, Phys. Rev. E. 81 (2010), p. 027301.

[34] J.P. Thibault, and L. Rossi, Experimental Modeling of Seawater Electromagnetic Flow Control, ERCOFTAC Bulletin on Turbulence Control 44 (2000), pp. 41-49.

[35] J.P. Thibault, and L. Rossi, Electromagnetic flow control: characteristic numbers and flow regimes of a wall-normal actuator, J. Phys. D: Appl. Phys. 36 (2003), p. 2559.

[36] G.A. Voth, T.C. Saint, G. Dobler, and J.P. Gollub, Mixing rates and symmetry breaking in twodimensional chaotic flow, Phys. Fluids 15 (2003), pp. 2560-2566.

[37] M.C. Jullien, P. Castiglione, and P. Tabeling, Experimental Observation of Batchelor Dispersion of Passive Tracers, Phys. Rev. Lett. 85 (2000), p. 3636.

[38] S.C. A. Figueroa F. Demiaux, and E. Ramos, Electrically driven vortices in a weak dipolar magnetic field in a shallow electrolytic layer, J. Fluid Mech. 641 (2009), pp. 245-261.

[39] R.A.D. Akkermans, A.R. Cieslik, L.P.J. Kamp, R.R. Trieling, H.J.H. Clercx, and G.J.F.v. Heijst, The three-dimensional structure of an electromagnetically generated dipolar vortex in a shallow fluid layer, Phys. Fluids 20 (2008), p. 116601. 
[40] D. Nosenchuck, and G. Brown, Discrete spatial control of wall shear stress in a turbulent boundary layer, in Near-Wall Turbulent Flows, edited by R. So C. Speziale, and B. Launder (Elsevier Science, New York) (1993), pp. 689-698.

[41] C. Henoch, and J. Stace, Experimental investigation of a salt water turbulent boundary layer modified by an applied streamwise magnetohydrodynamic body force, Phys. Fluids 7 (1995), pp. 1371-1383.

[42] K.S. Breuer, J. Park, and C. Henoch, Actuation and control of a turbulent channel flow using Lorentz forces, Phys. Fluids 16 (2004), pp. 897-907.

[43] J.H. Lee, and H. Sung, "Response of a spatially developing turbulent boundary layer to a spanwise oscillating electromagnetic force", Journal of Turbulence 6 (2005), pp. 1-15.

[44] T. Weier, G. Gerbeth, G. Mutschke, O. Lielausis, and G. Lammers, Control of Flow Separation Using Electromagnetic Forces, Flow, Turbulence and Combustion 71 (2003), pp. 5-17 10.1023/B:APPL.0000014922.98309.21.

[45] L. Rossi, and J.P. Thibault, Investigation of wall normal electromagnetic actuator for seawater flow control, Journal of Turbulence 3 (2002), p. N5.

[46] S. Kenjeres, J. Verdoold, M. Tummers, K. Hanjalic, and C. Kleijn, Numerical and experimental study of electromagnetic driven vortical flows, ETMM7 (2008), p. 6.

[47] S. Kenjeres, Electromagnetic enhancement of turbulent heat transfer, Phys. Rev. E 78 (2008), p. 066309.

[48] A. Wolf, M. Brown, and P. Prentiss, Concentrative properties of aqueous solutions: conversion tables, CRC Handbook of Chemestry and Physics, CRC Press (1982).

[49] R. Moreau "Magnetohydrodynamics", Kluwer , 1990.

[50] S. Lardeau, S. Ferrari, and L. Rossi, Three-dimensional direct numerical simulation of electromagnetically driven multiscale shallow layer flows: Numerical modeling and physical properties, Phys. Fluids 20 (2008), p. 127101.

[51] S. Ferrari, and L. Rossi, "Particle tracking velocimetry and accelerometry (PTVA) measurements applied to quasi-two-dimensional multi-scale flows", Exp. Fluids 44 (2008), pp. 873-886.

[52] G. Akoun, and J. Yonnet, 3D analytical calculation of the forces exerted between two cuboidal magnets, IEEE Transaction on magnetics 20 (1984), pp. 1962-1964.

[53] J. Salazar, and L. Collins, Two-Particle Dispersion in Isotropic Turbulent Flows, Annu. Rev. Fluid. Mech. 41 (2009), pp. 405-432.

[54] G. Batchelor, Small-scale variation of convected quantities like temperature in turbulent fluid, J. .Fluid Mech. 437 (1959), pp. 113-133.

[55] P. Davidson Turbulence, an introduction for scientists and engineers, Oxford University Press, 2004. 

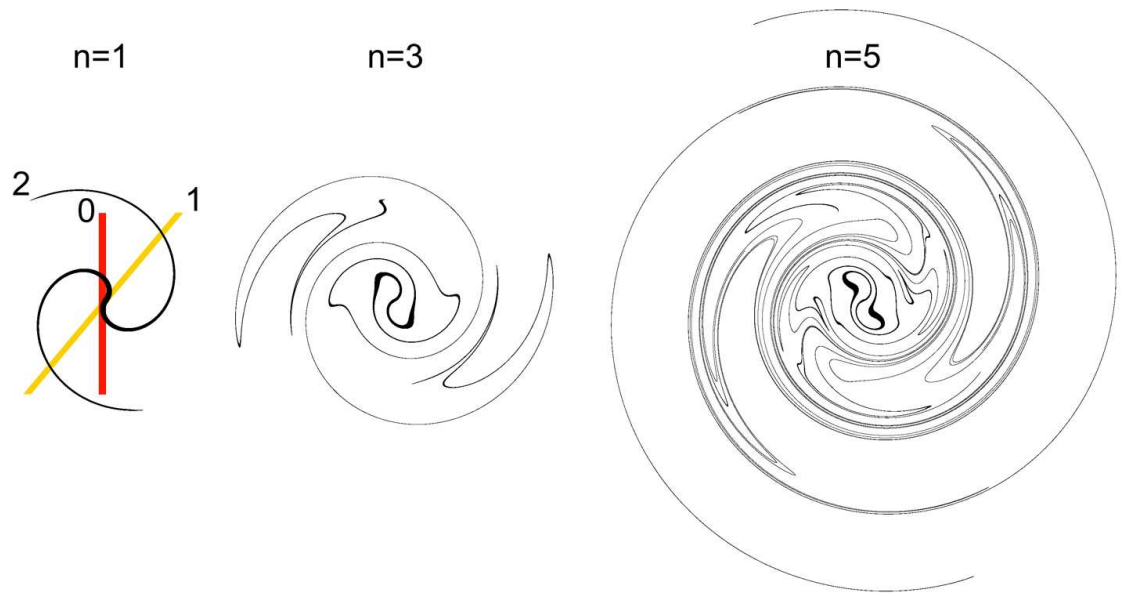

Figure 1. Stretching of a coloured band via cyclic combinations of shear and lamination. The building of these cycles is illustrated for the first iteration, $n=1$. Also, the whorl used to trigger lamination has a constant angular velocity.

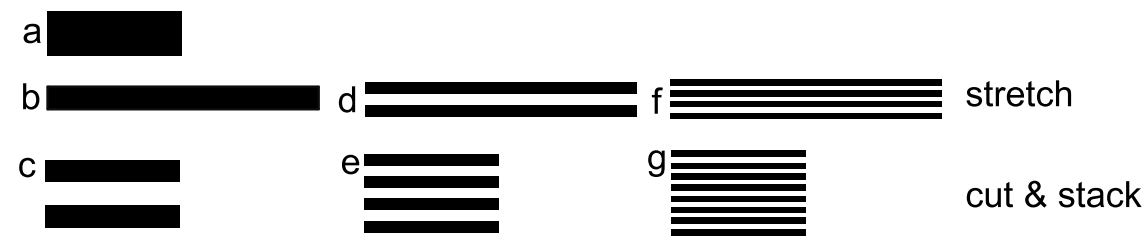

Figure 2. Successive stretching, cutting and stacking of material bands usually called a baker map. The material band total length is growing exponentially within a confined domain. The domain's length-scale is smaller than the total length of the band after only 2 iterations.

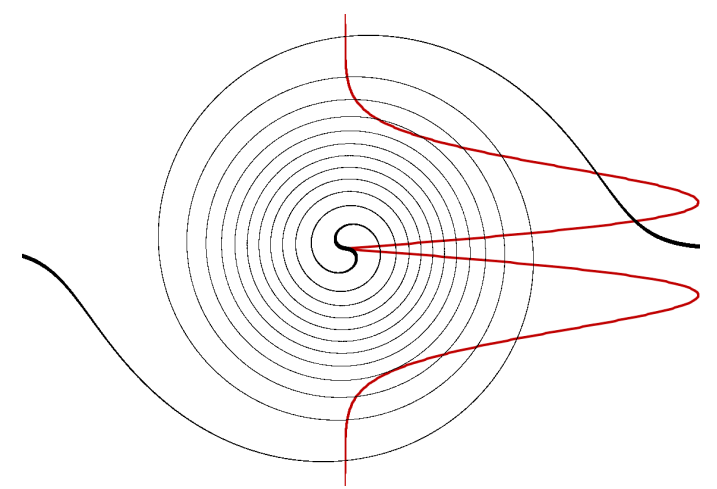

Figure 3. Lamination within a whorl of angular velocity $\dot{\theta}=\dot{\theta}_{0} \exp \left(1-(r / R)^{2}\right)$ after 6 revolutions. The black and red lines respectively illustrate the whorl and $\partial \dot{\theta} / \partial r$.

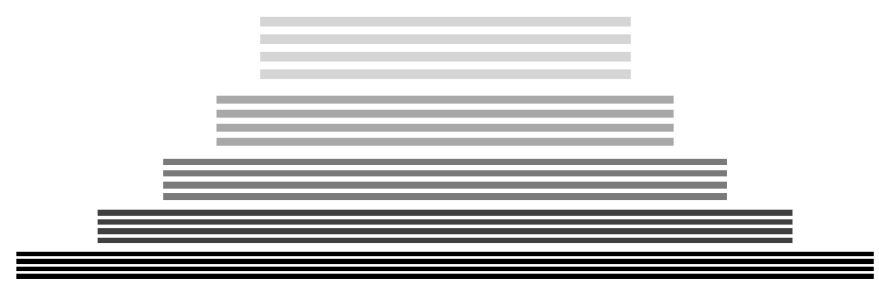

Figure 4. Evolution of a striated structure in a pure strain region. The temporal evolutions are indicated by varying the bands' colour from from light grey (initial distribution) to black. 


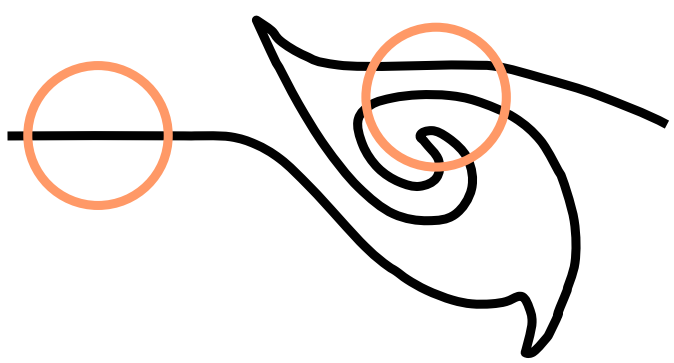

Figure 5. Illustration of a line which has been stirred (cat's eye flow structure) during one eddy turnover time. The circles represent the measure of lamination along the material line.

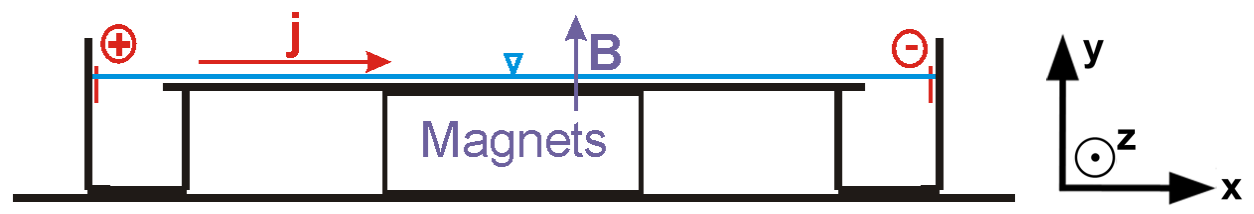

Figure 6. Side view of the electromagnetic mixer rig

(a)

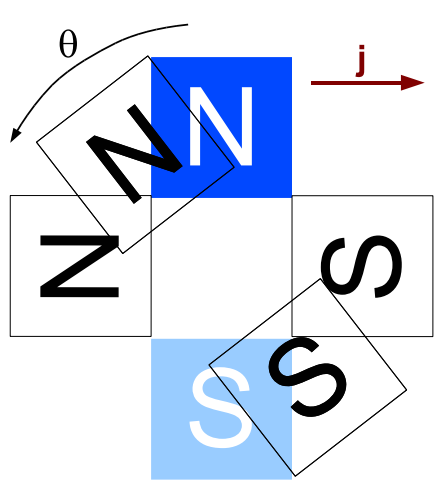

(b)

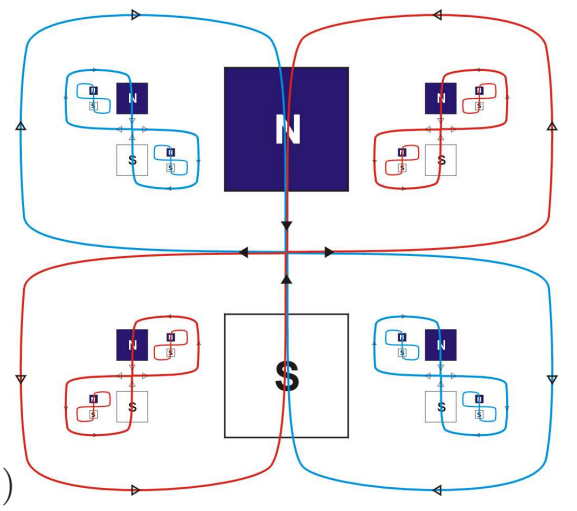

Figure 7. Distribution of permanent magnets underneath the wall. one- (a) and multi- (b) scale configurations 
$0^{\circ}$

$15^{\circ}$

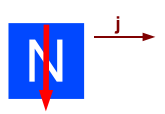

本

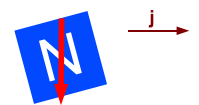

本

$90^{\circ}$

(a)
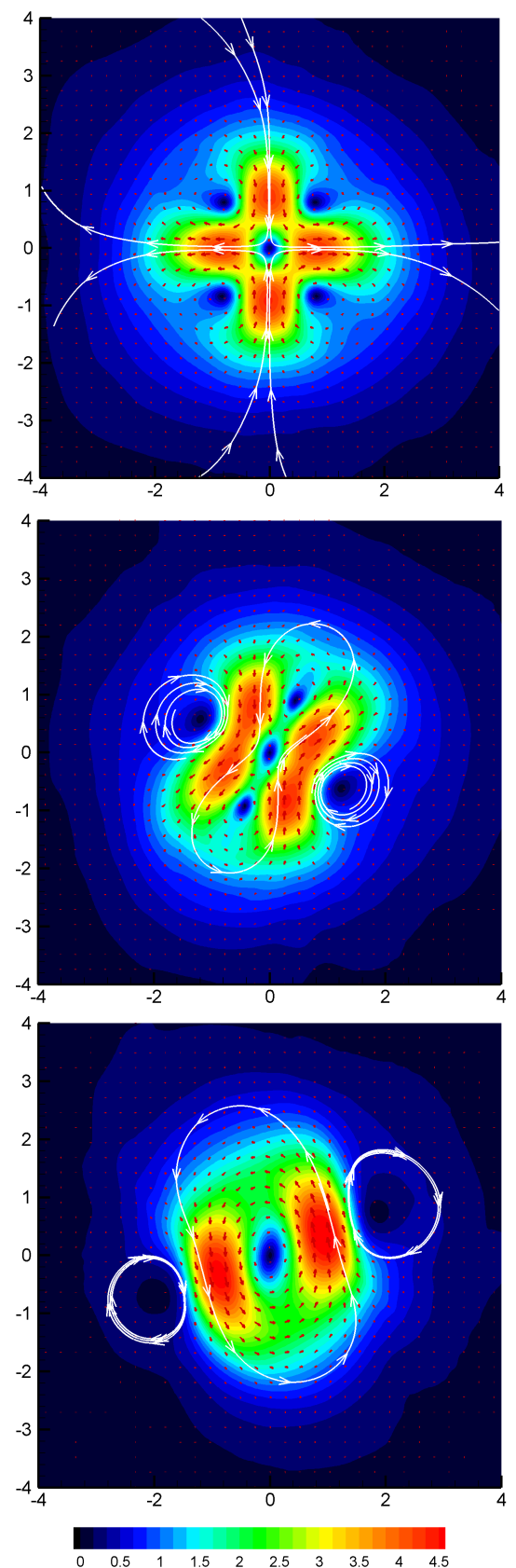

(b)
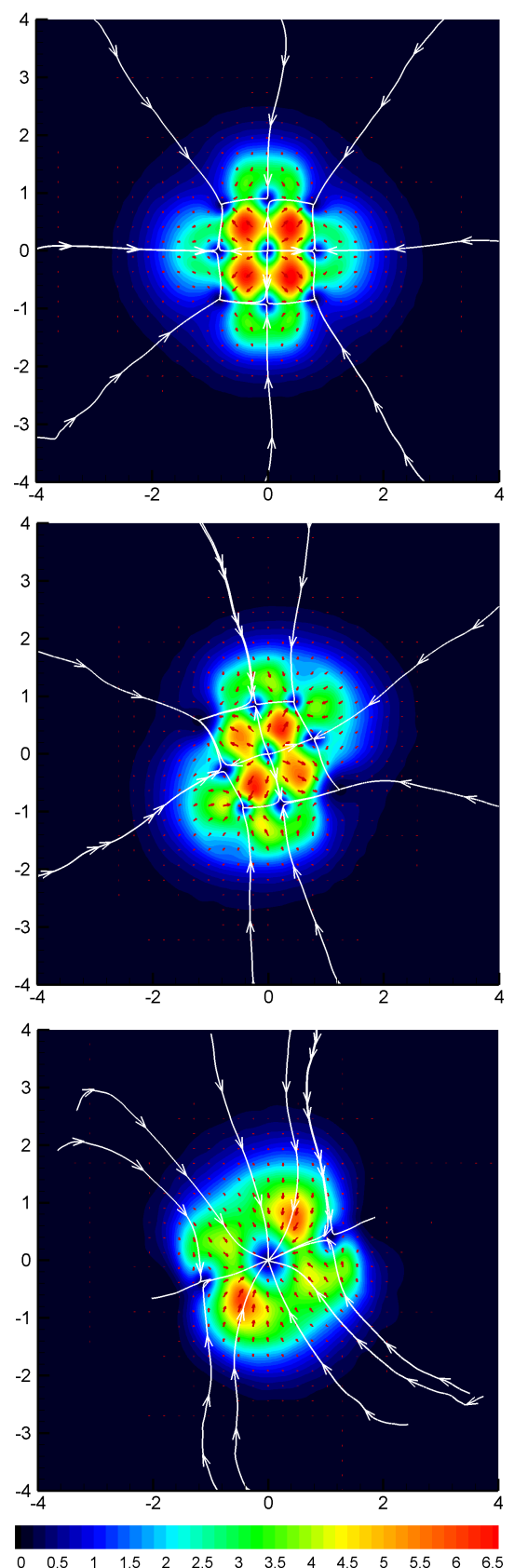

(c)

Figure 8. (a) Schematics of the different forcing configurations. The arrows indicate the direction of the electrical current $\mathbf{j}$ and of the main forces pumping the flow. (b) velocity fields, $\mathbf{u} / u_{r m s}$ (c) acceleration fields, $\mathbf{a} / a_{r m s}$. 


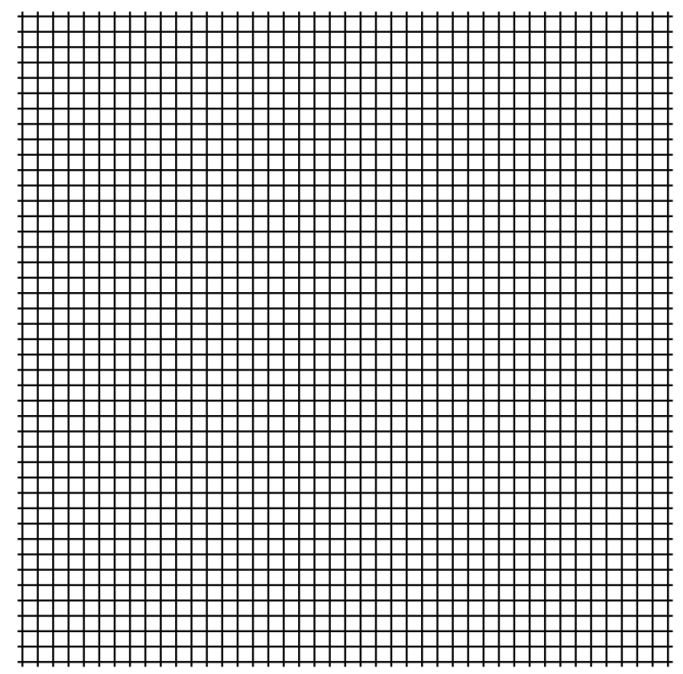

initial grid

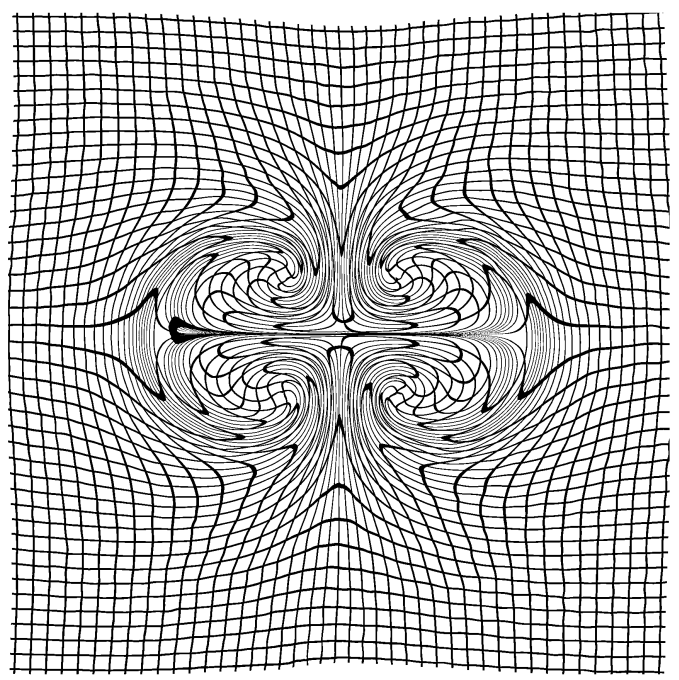

$0^{\circ}$

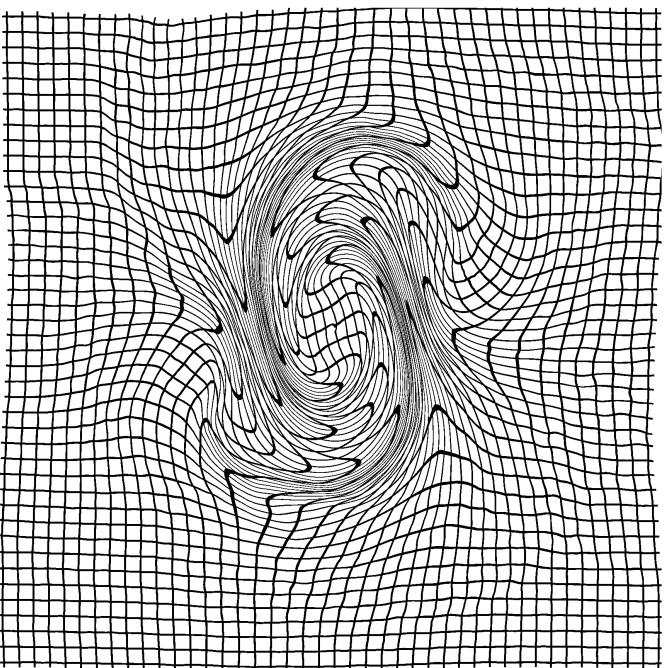

Figure 9. Illustration of a regular grid deformation over the entire PIV-PTVA domain. These visualisations

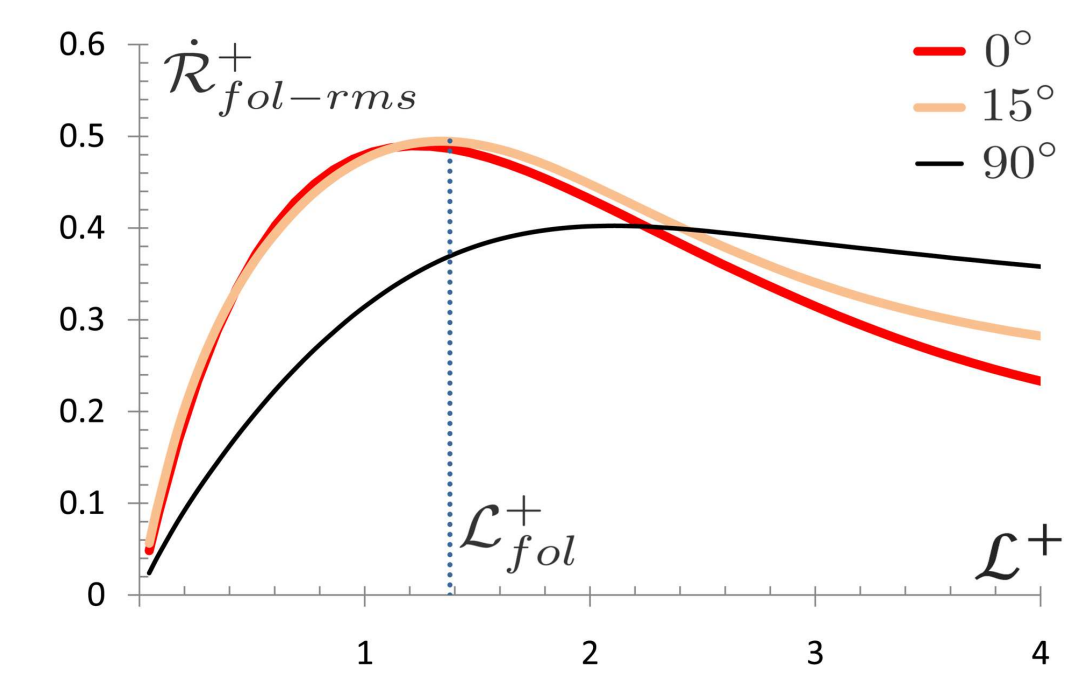

Figure 10. Evolution of lamination rate intensity $\dot{R}_{\text {fol-rms }}^{+}$with filtering length-scale $\mathcal{L}^{+}$.

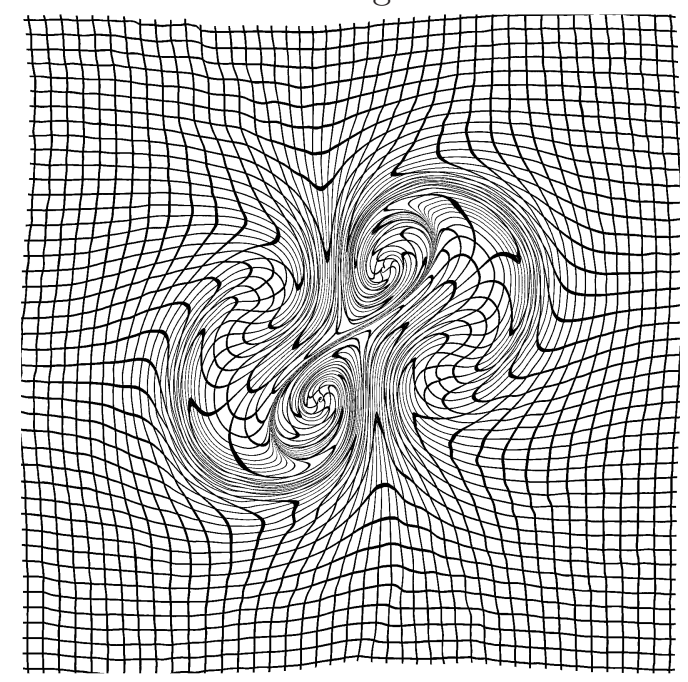

$15^{\circ}$

$90^{\circ}$

are performed by tracking fluid elements within the velocity fields during $t^{+} \simeq 4.9$. 

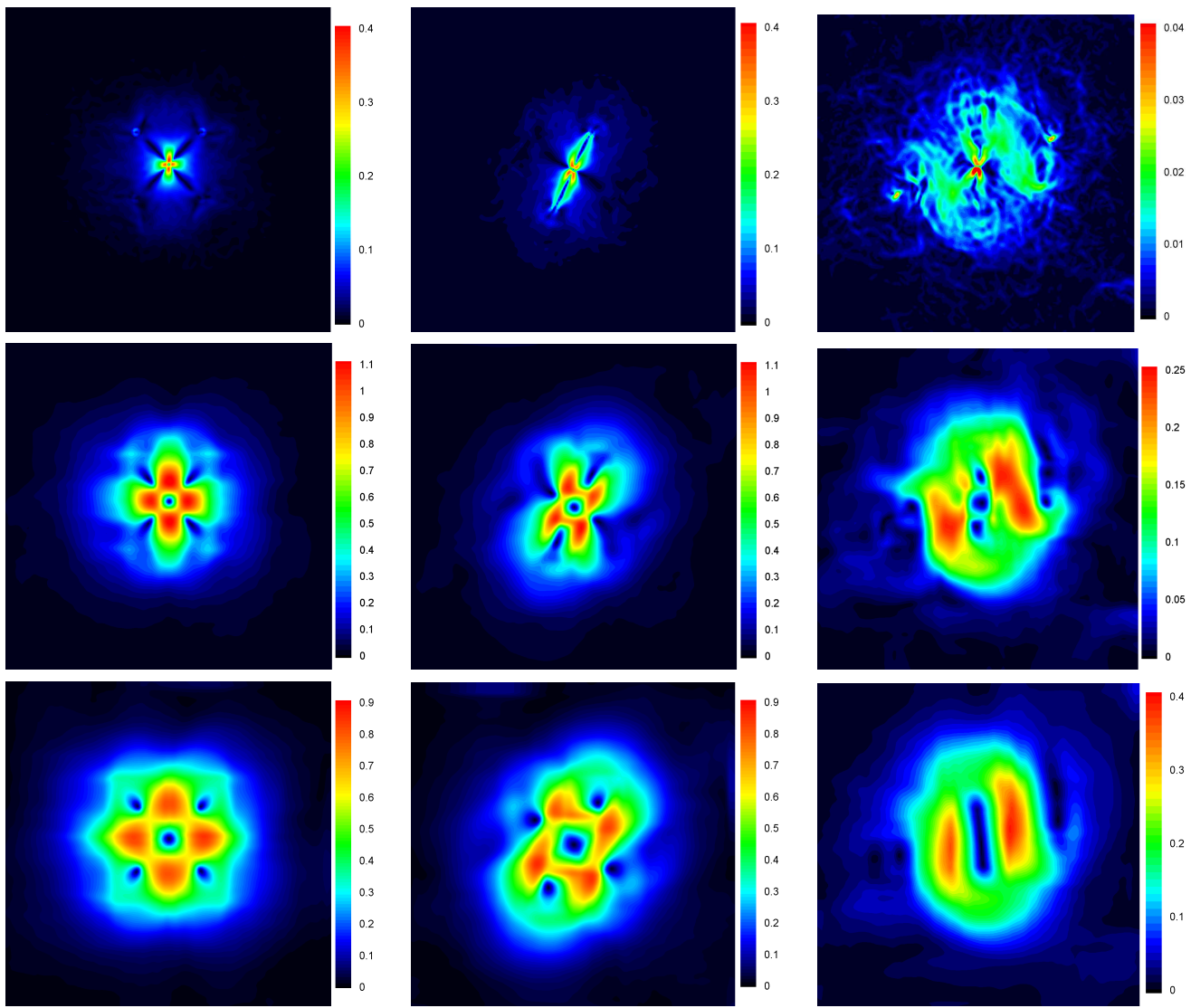

$0^{\circ}$

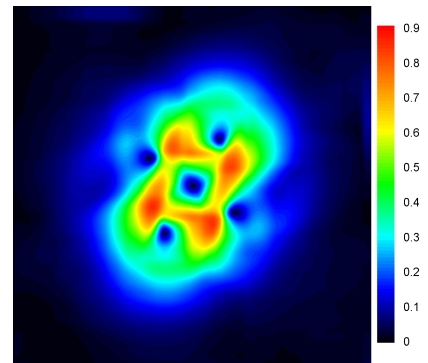

$15^{\circ}$

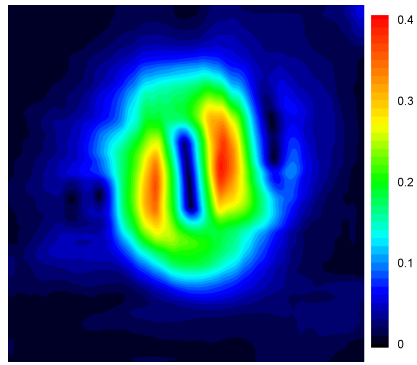

$90^{\circ}$

Figure 11. Lamination rate intensity $\dot{R}_{\text {fol }}^{+}$, for the three flow configurations, for (from top to bottom) (a) $\mathcal{L}^{+}=0.086$, (b) $\mathcal{L}^{+}=0.69$ and (c) $\mathcal{L}^{+}=1.38$. Same domain as Fig. 8.

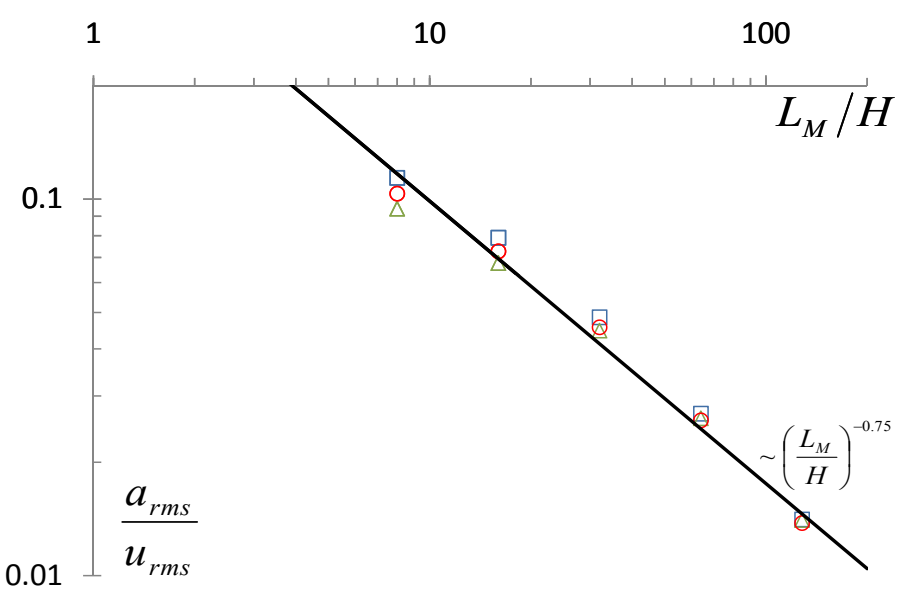

Figure 12. Evolution of the characteristic frequency, $a_{r m s} / u_{r m s}$, versus $L_{M} / H$. The line indicates $a_{r m s} / u_{r m s} \sim\left(L_{M} / H\right)^{-3 / 4}$. 


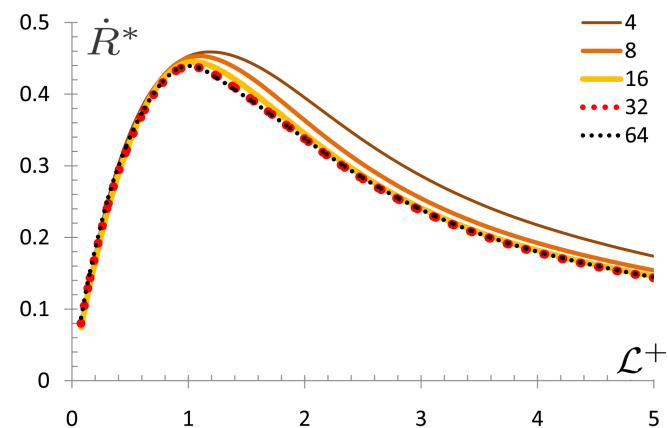

(a)

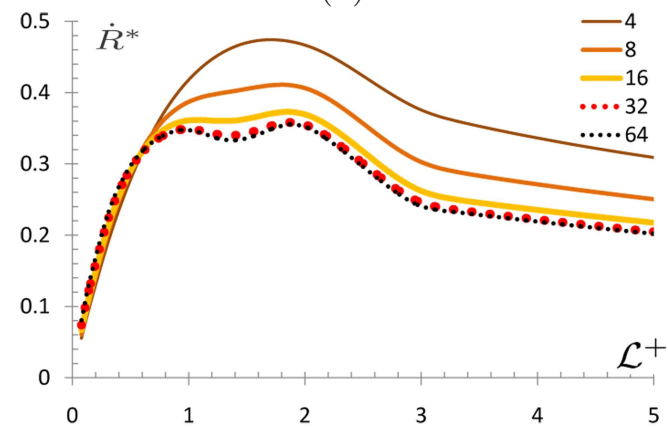

(c)

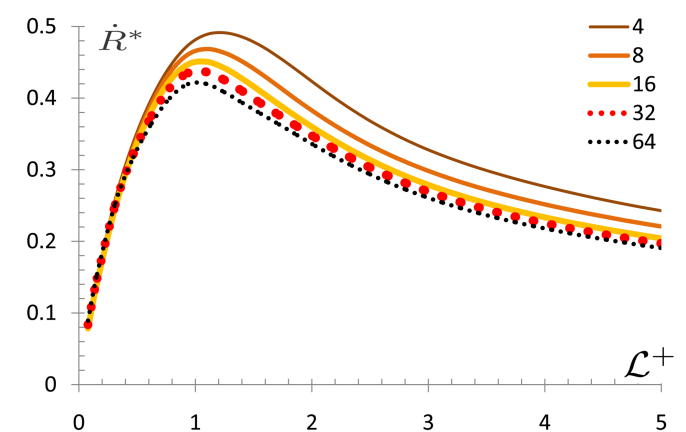

(b)

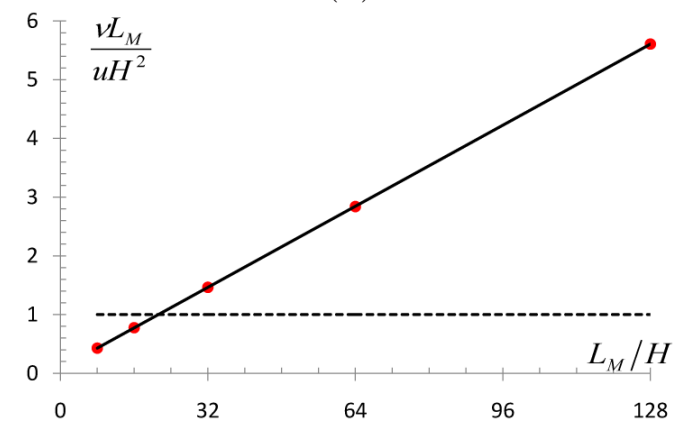

(d)

Figure 13. Evolution of the dimensionless lamination rate intensity $\dot{R}^{*}=u_{r m s} \dot{R}_{f o l-r m s} / a_{r m s}$ with length-scale $\mathcal{L}^{+}$. The size of the magnets varies from $4 \mathrm{~cm}$ to $64 \mathrm{~cm}$, for (a) $0^{\circ}$, (b) $15^{\circ}$, (c) $90^{\circ}$. (d) gives the ratio of the turnover time $L_{M} / u$ divided by the viscous damping time $H^{2} / \nu$. u is taken as the rms over the centre domain of size $5 L_{M} \times 5 L_{M}$. The solid line corresponds to the linear trend $\frac{\nu L_{M}}{u H^{2}}=0.0431 \frac{L_{M}}{H}+0.0834$.
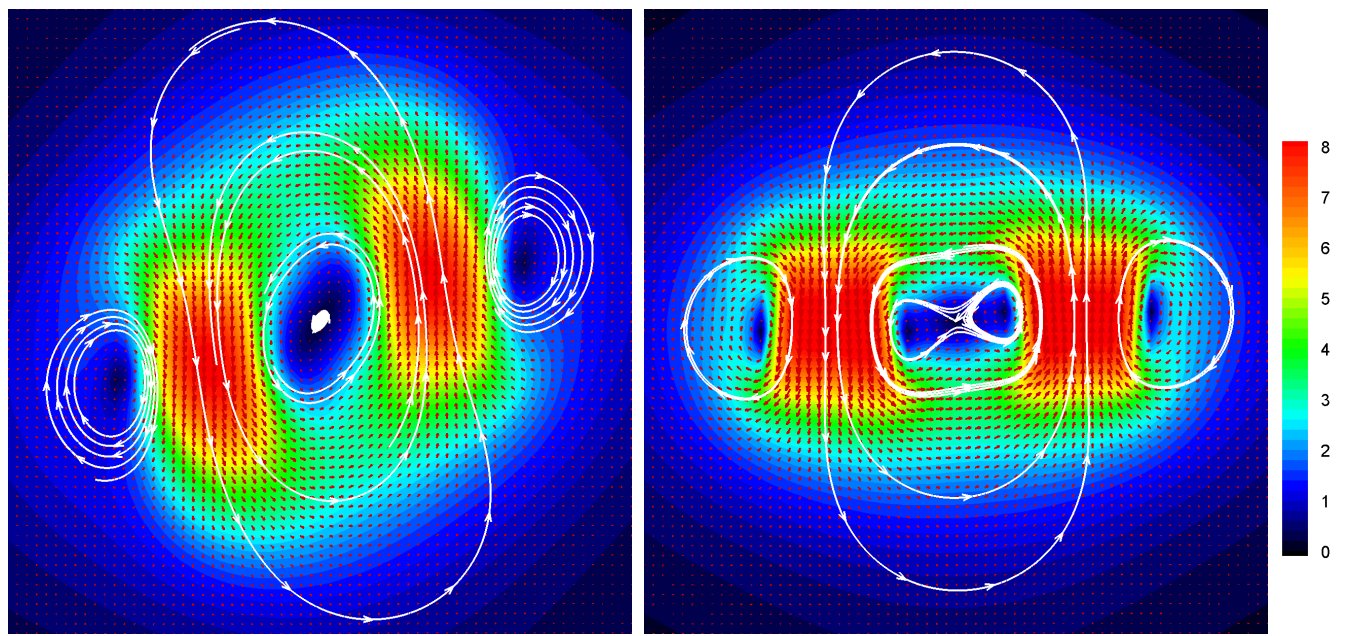

Figure 14. Velocity fields $\mathbf{u} / u_{r m s}$ for the orientation $90^{\circ}$ and two different magnet sizes: $4 \mathrm{~cm}$ and $64 \mathrm{~cm}$. Streamlines are plotted in white. Only the centre domain of size $5 L_{M} \times 5 L_{M}$ is plotted. 


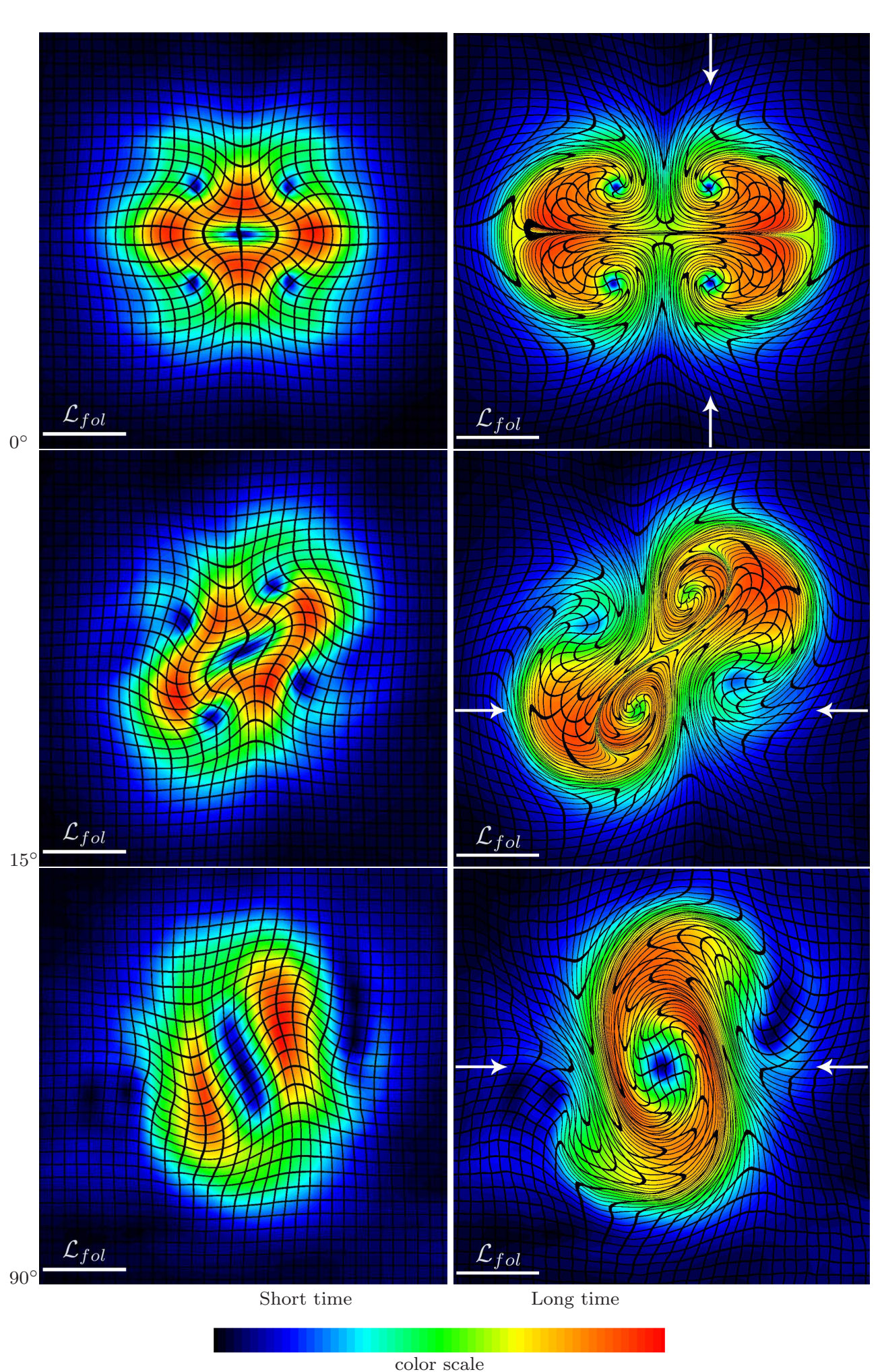

Figure 15. Grid deformation superimposed onto color maps representing the time average lamination rate computed for $\mathcal{L}^{+}=1.38$, for (left) $t^{+} \simeq 0.61$ and (right) $t^{+}=4.9$. Zeros are shown in black and red

indicates maximum values. Color scales maxima, from top left to bottom right, are : 0.9, 0.6, 0.9, 0.6, 0.4,
0.3 . 0.3 . 


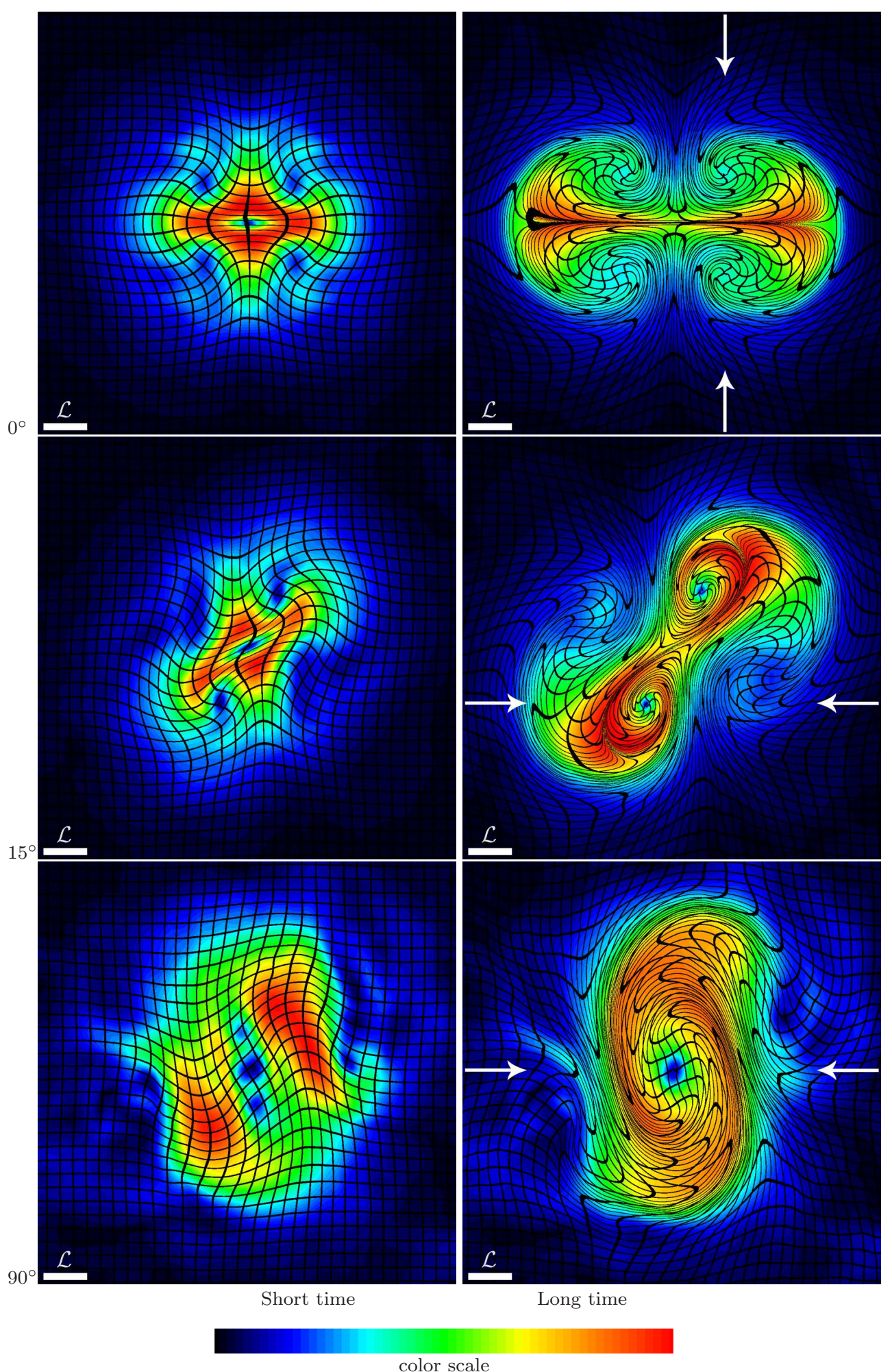

Figure 16. Grid deformation superimposed onto color maps representing the time average lamination rate computed for $\mathcal{L}^{+}=0.69$, for (left) $t^{+} \simeq 0.61$ and (right) $t^{+}=4.9$. Zeros are shown in black and red indicates maximum values. Color scales maxima, from top left to bottom right, are : 1.1, 0.7, 1.1, 0.6, 0.25, 0.2 .

$0^{\circ}$

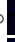

Long time

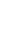



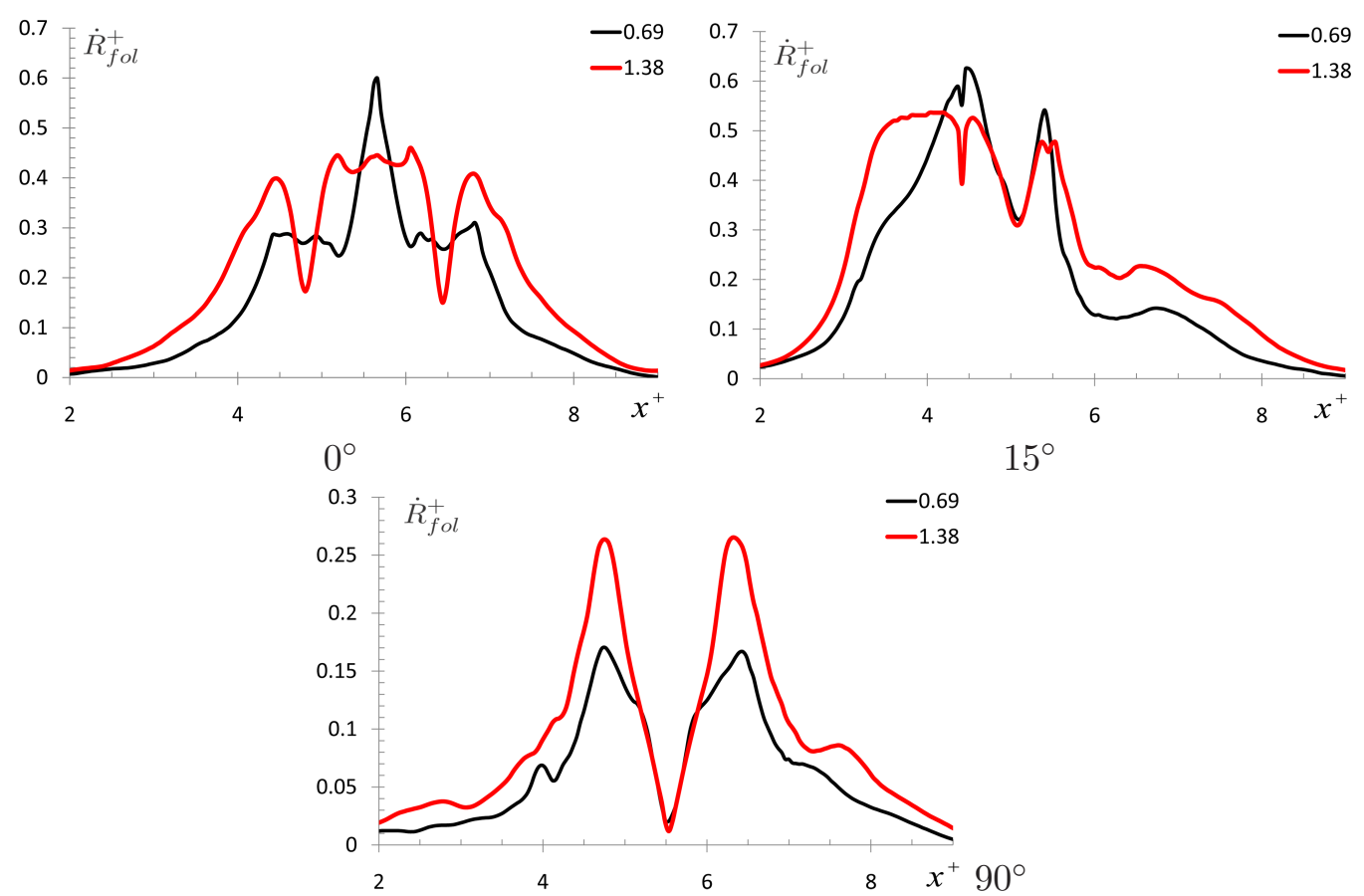

Figure 17. Profiles of the time-average lamination rate, $\dot{R}_{f o l}^{+}$, extracted from Fig. 15 and 16 along the directions indicated by white arrows. Black and red lines correspond to $\mathcal{L}^{+}=0.69$ and $\mathcal{L}^{+}=1.38$, respectively. 

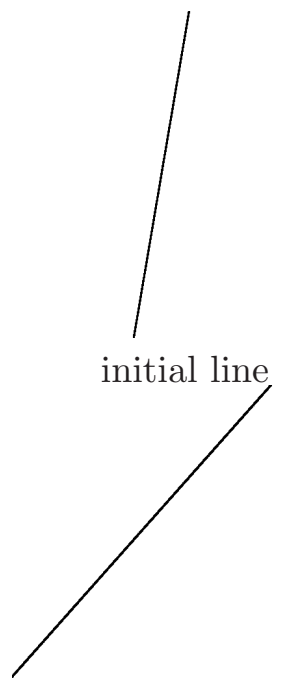

initial line
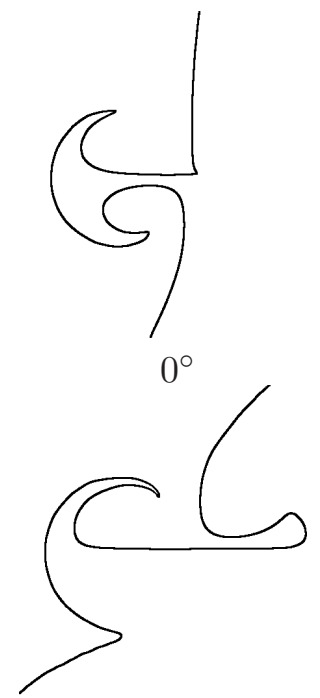

$0^{\circ}$
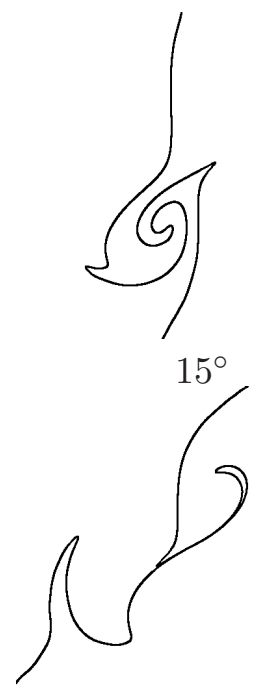

$15^{\circ}$

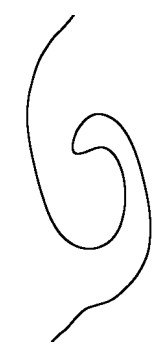

$90^{\circ}$

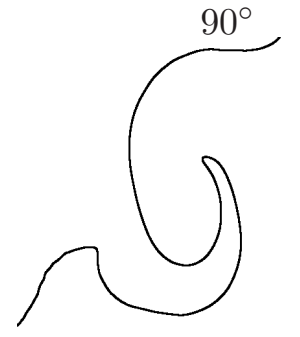

$90^{\circ}$

Figure 18. Examples of lines tracked in time. Initial lines (on the left) and tracked lines at $t^{+}=4.9$ for the three flow configurations, $0^{\circ}, 15^{\circ}, 90^{\circ}$.
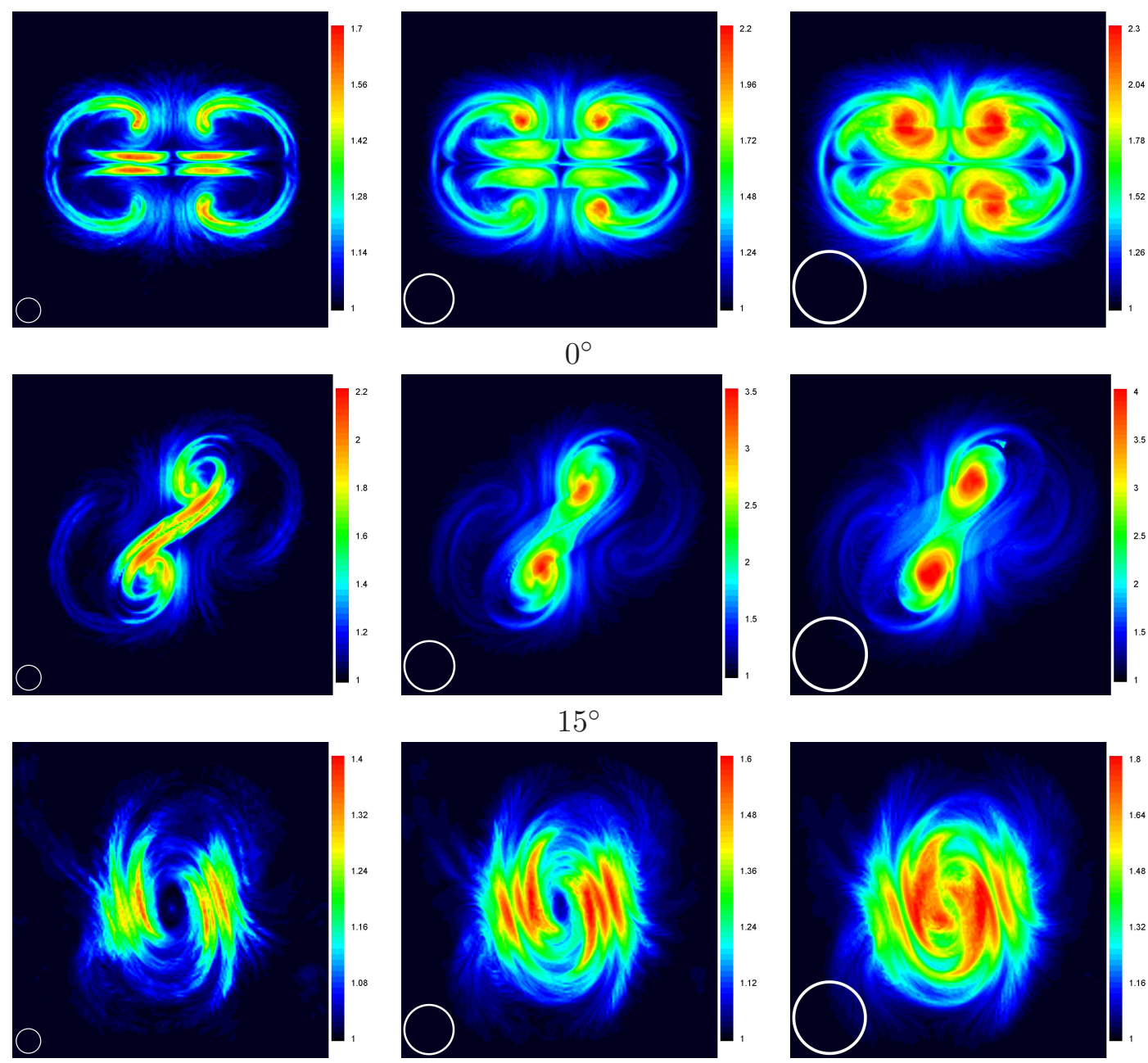

$90^{\circ}$

Figure 19. Lamination measure maps for $0^{\circ}, 15^{\circ}, 90^{\circ}$, and for three different diameters $\phi$ (indicated by circles on bottom left corner of each plot). Their diameters are: $\phi^{+}=0.5,1$ and 1.5 . 


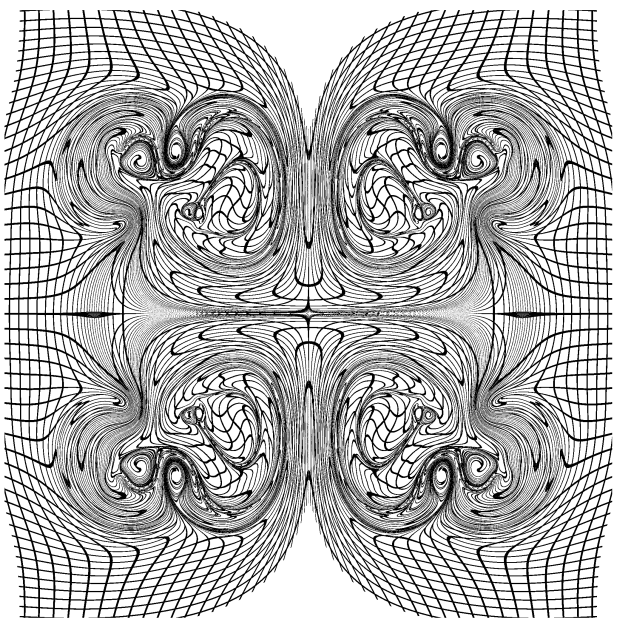

(a)

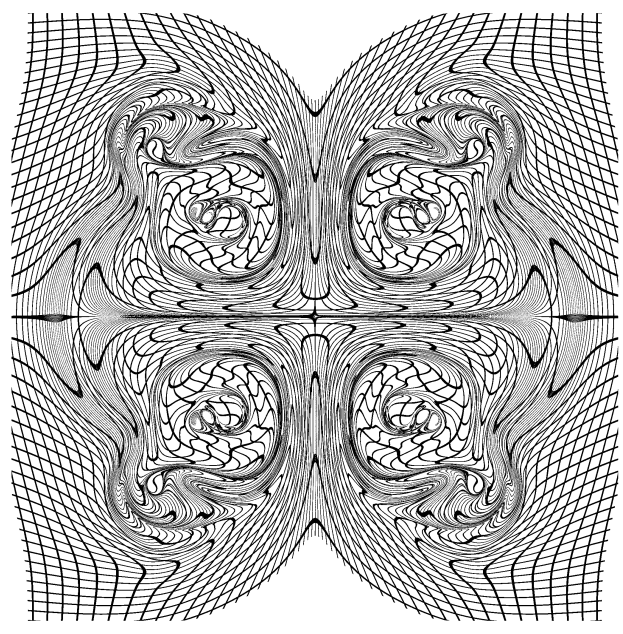

(b)

Figure 20. Grid deformation for the multi-scale flow configurations, for two different Reynolds numbers: (a) $R e=3$ and (b) $R e=25$. Movies "grid_Re3.avi" and "grid_Re25.avi" gives the animation associated with these visualisations.

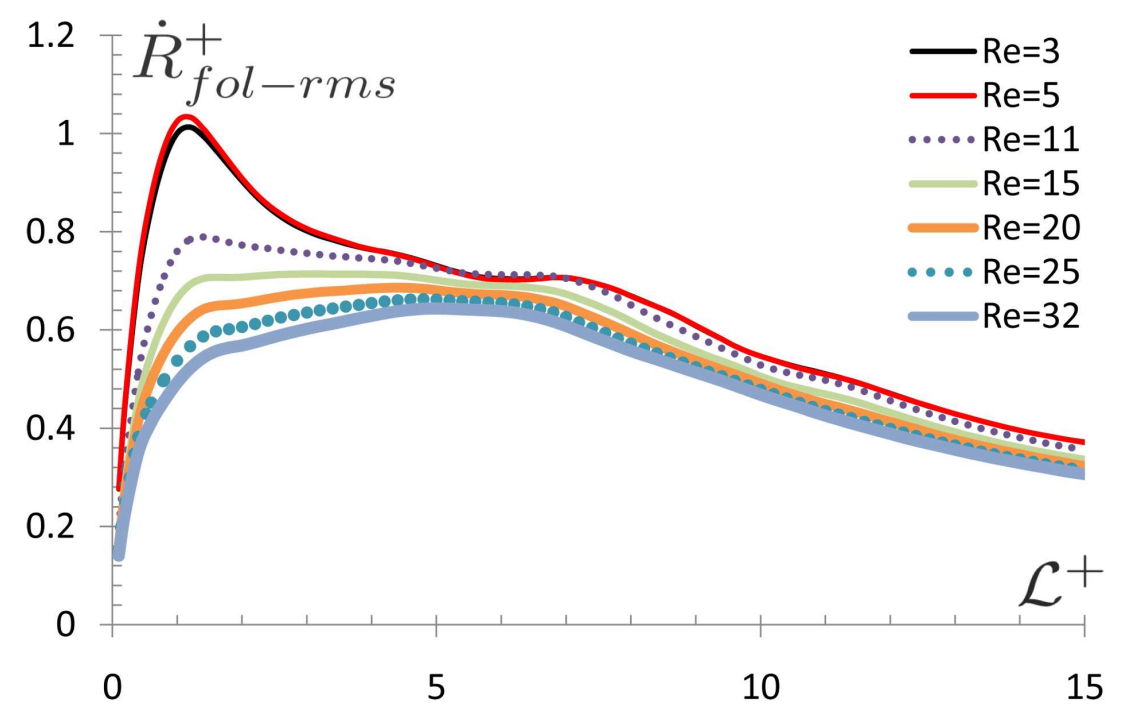

Figure 21. Evolution of the lamination rate, $\dot{R}_{\text {fol-rms }}^{+}$as a function of $\mathcal{L}^{+}$, for different Reynolds numbers. 

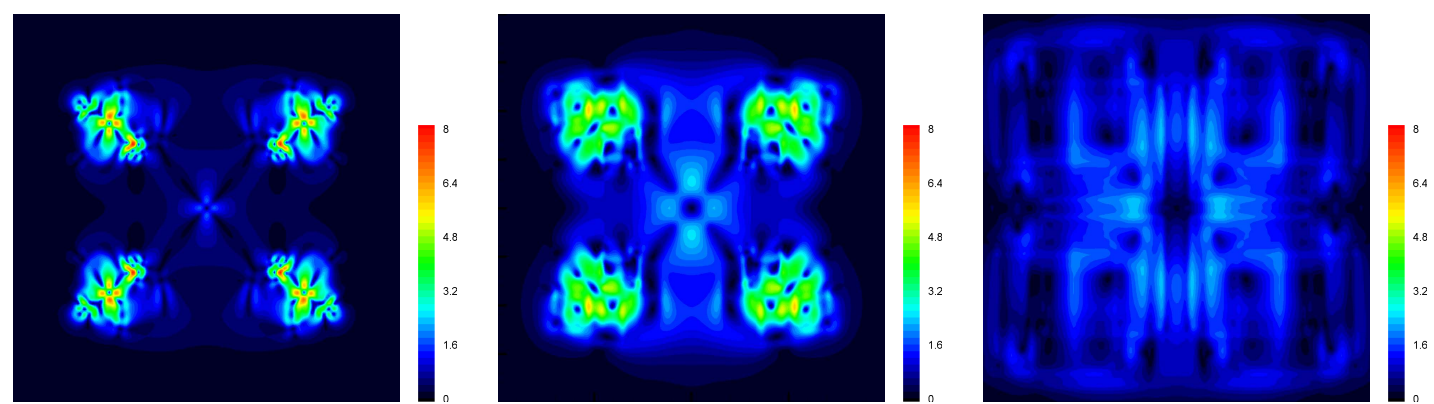

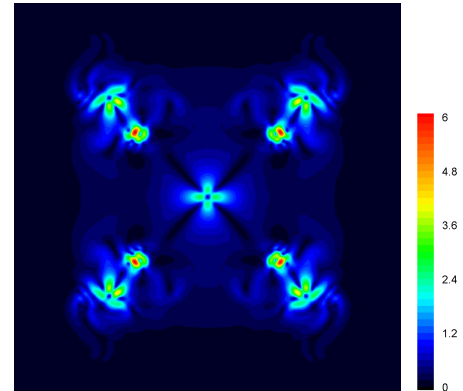

(a)

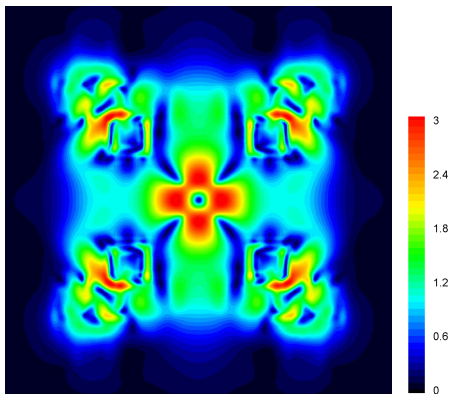

(b)

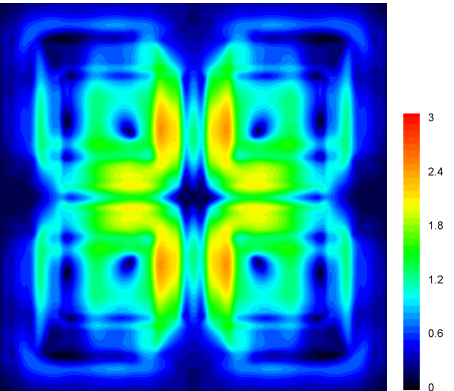

(c)

Figure 22. Lamination rate maps for the multi-scale flows, for (top row) $R e=3$ and (bottom row) $R e=25$. The filtering length-scale are: (a) $\mathcal{L}^{+}=0.4$, (b) $\mathcal{L}^{+}=1.6$ and (c) $\mathcal{L}^{+}=6$ 


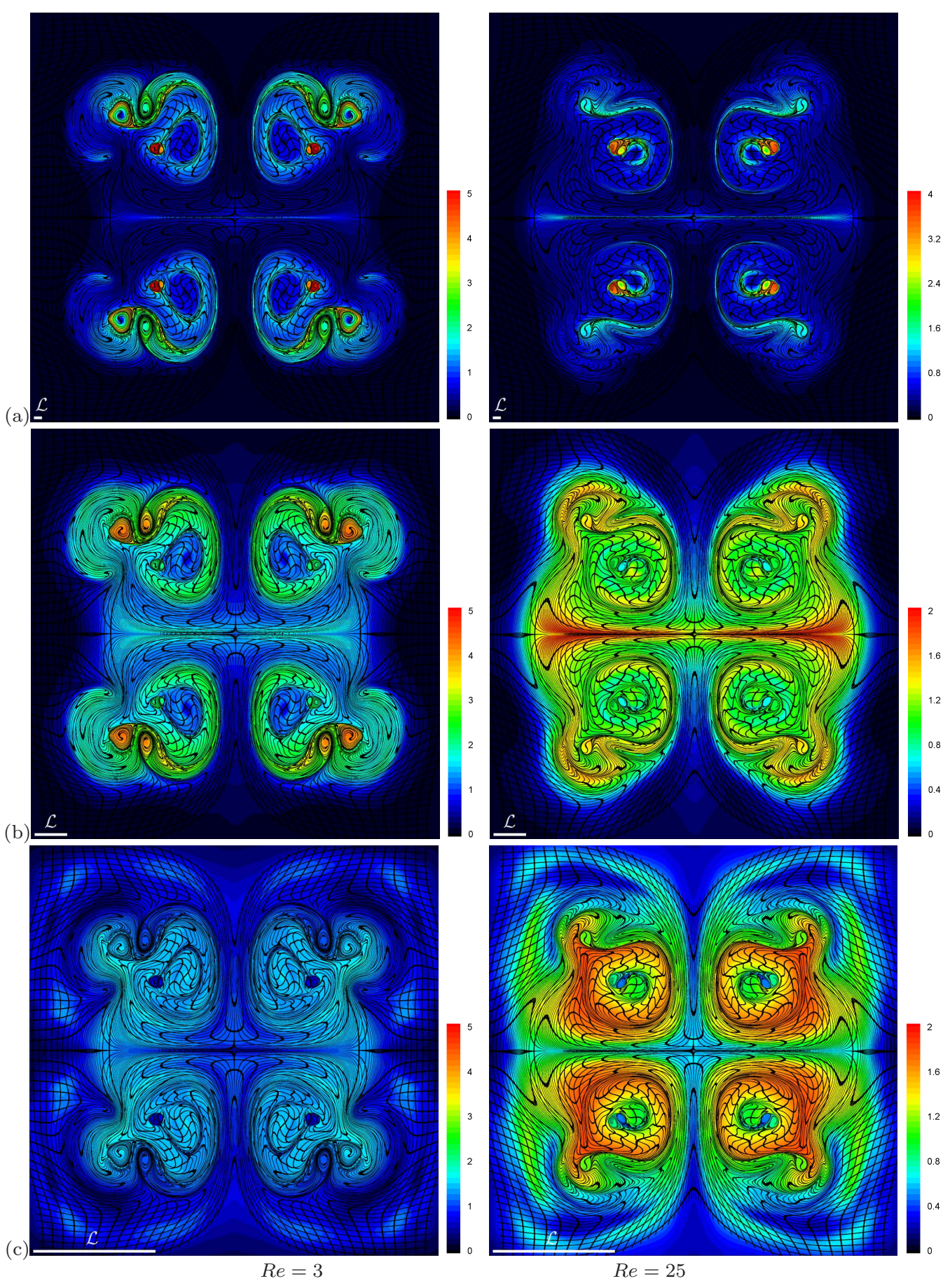

Figure 23. Grid deformation superimposed onto color maps representing the time average lamination rate computed for the length-scales: (a) $\mathcal{L}^{+}=0.4$, (b) $\mathcal{L}^{+}=1.6$ and (c) $\mathcal{L}^{+}=6$. Left column is for $\operatorname{Re}=3$ and right column shows maps for $R e=25$. 

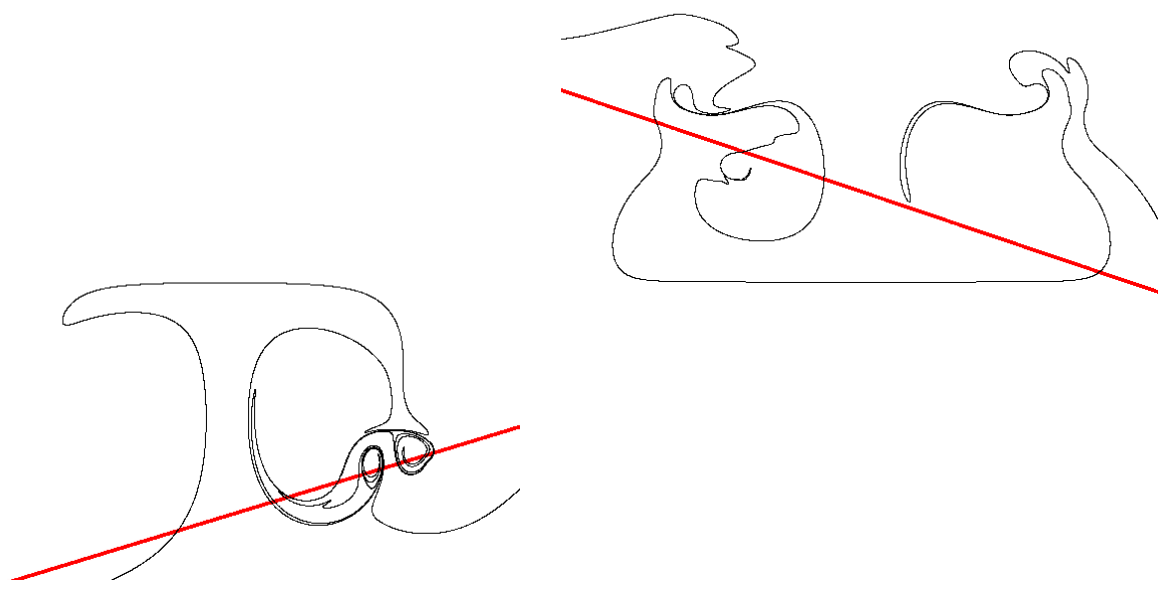

$$
R e=3
$$

$$
R e=25
$$

Figure 24. Illustration of tracked lines for $R e=3$ and $R e=25$. Snapshot after one turn-over time. Red line indicates the initial position of the tracked line.

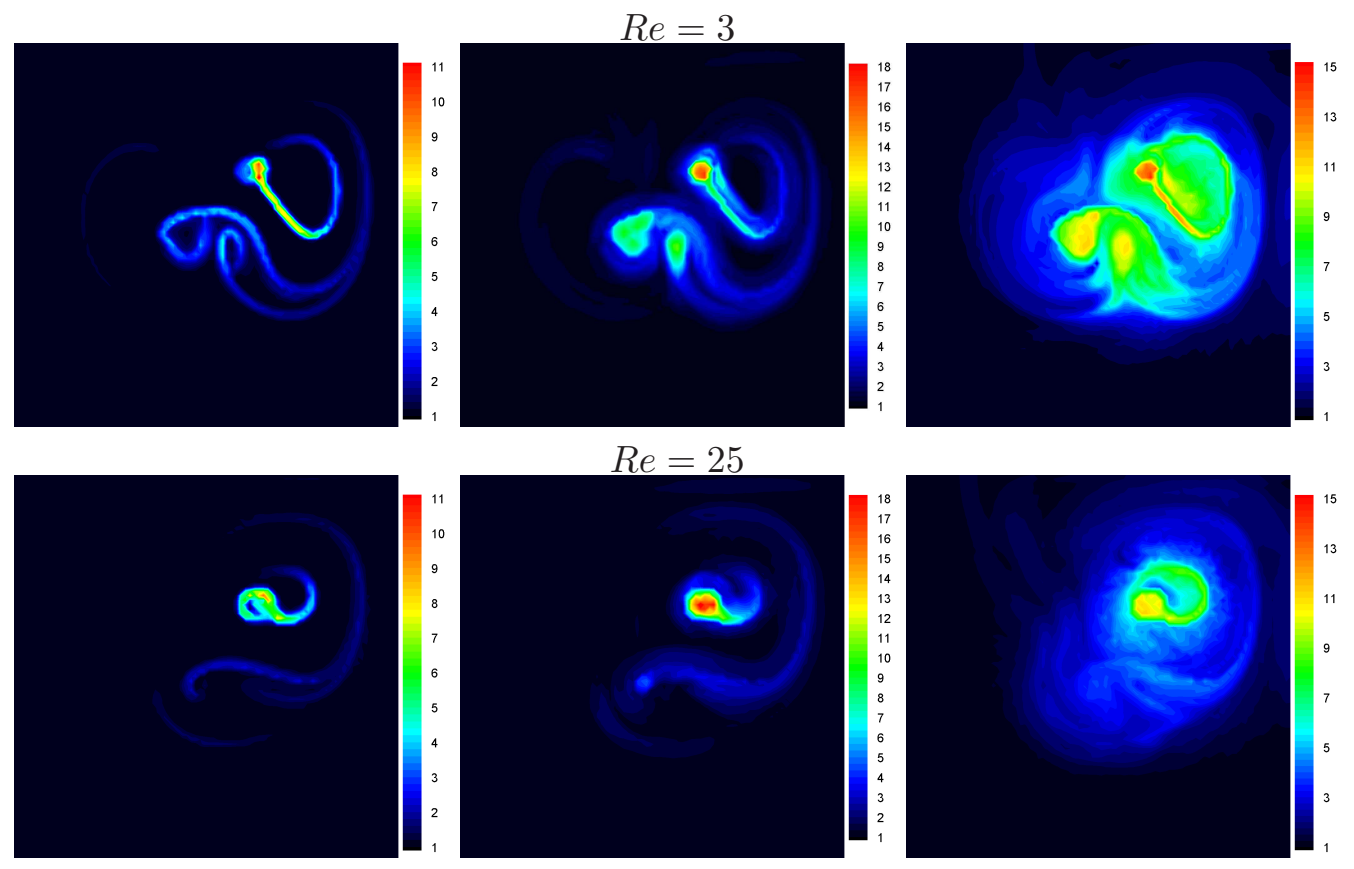

Figure 25. Measure of lamination within circles of diameters $\phi^{+}=0.25, \phi^{+}=1$ and $\phi^{+}=4$ for the Reynolds numbers $R e=3$ and $R e=25$. The measures are performed after one turnover time $4 L_{M} / u_{r m s}$ in both cases. The corresponding dimensionless times are $t^{+}=15.6$ for $R e=3$ and $t^{+}=6.17$ for $R e=25$. Only a quarter of the domain shown on Fig. 23 is represented, for clarity. 


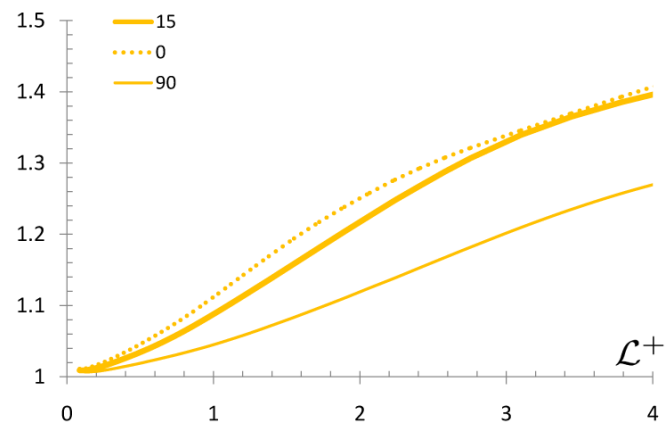

(a)

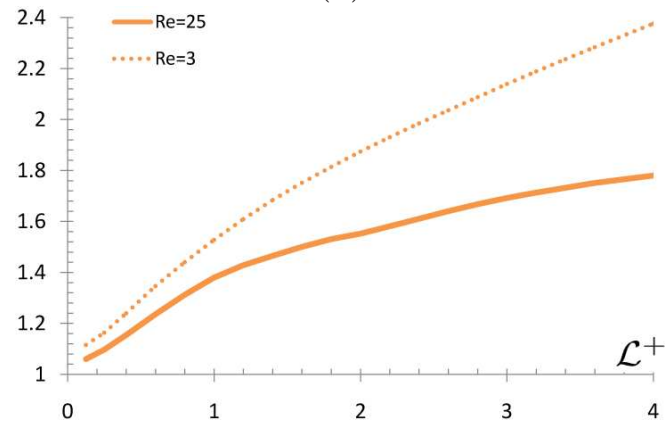

(c)

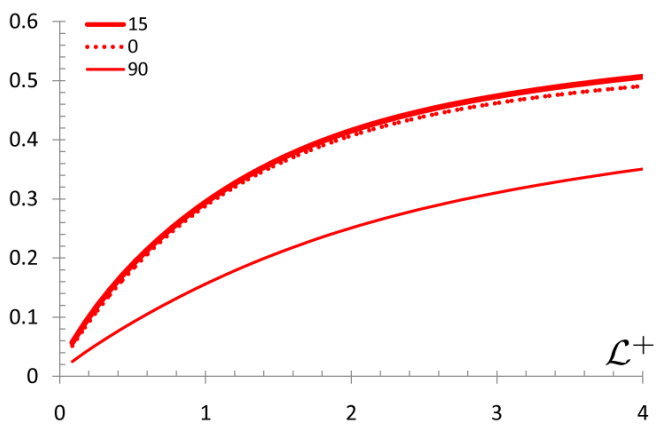

(b)

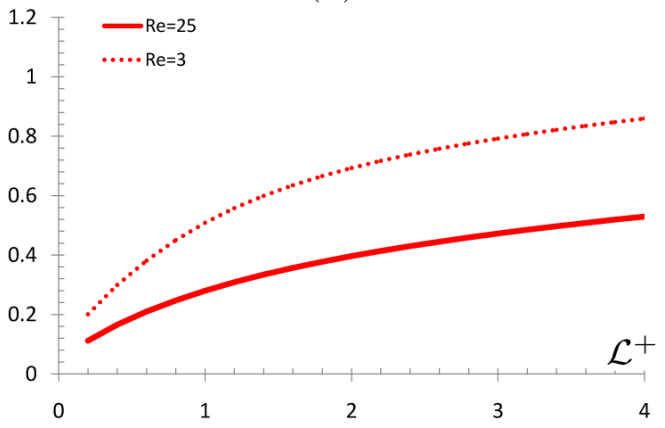

(d)

Figure 26. Comparions between $(\mathrm{a}, \mathrm{c})$ measure of lamination and $(\mathrm{b}, \mathrm{d})$ relative lamination increment $\mathcal{G}$ (equation 10). (Top row) Mono-scale configuration for the three magnets orientations, at $t^{+}=4.9$. (Bottom row) Multi-scale configurations for $R e=3$ and $R e=25$, comparions performed after one turnover time, $4 L_{M} / u_{r m s}$, i.e. $t^{+}=15.6$ for $R e=3$ and $t^{+}=6.17$ for $R e=25$. 